\title{
Underlying mechanisms of transient luminous events: a review
}

\author{
V. V. Surkov ${ }^{1}$ and M. Hayakawa ${ }^{2}$ \\ ${ }^{1}$ Moscow Engineering Physics Institute (MEPhI), Research Nuclear University of the Russian Federation, Moscow, Russia \\ ${ }^{2}$ Advanced Wireless Communications Research Center, The University of Electro-Communications, Chofu Tokyo, Japan \\ Correspondence to: M. Hayakawa (hayakawa@whistler.ee.uec.ac.jp)
}

Received: 16 February 2012 - Revised: 24 July 2012 - Accepted: 30 July 2012 - Published: 17 August 2012

\begin{abstract}
Transient luminous events (TLEs) occasionally observed above a strong thunderstorm system have been the subject of a great deal of research during recent years. The main goal of this review is to introduce readers to recent theories of electrodynamics processes associated with TLEs. We examine the simplest versions of these theories in order to make their physics as transparent as possible. The study is begun with the conventional mechanism for air breakdown at stratospheric and mesospheric altitudes. An electron impact ionization and dissociative attachment to neutrals are discussed. A streamer size and mobility of electrons as a function of altitude in the atmosphere are estimated on the basis of similarity law. An alternative mechanism of air breakdown, runaway electron mechanism, is discussed. In this section we focus on a runaway breakdown field, characteristic length to increase avalanche of runaway electrons and on the role played by fast seed electrons in generation of the runaway breakdown. An effect of thunderclouds charge distribution on initiation of blue jets and gigantic jets is examined. A model in which the blue jet is treated as upward-propagating positive leader with a streamer zone/corona on the top is discussed. Sprite models based on streamer-like mechanism of air breakdown in the presence of atmospheric conductivity are reviewed. To analyze conditions for sprite generation, thunderstorm electric field arising just after positive cloudto-ground stroke is compared with the thresholds for propagation of positively/negatively charged streamers and with runway breakdown. Our own estimate of tendril's length at the bottom of sprite is obtained to demonstrate that the runaway breakdown can trigger the streamer formation. In conclusion we discuss physical mechanisms of VLF (very low frequency) and ELF (extremely low frequency) phenomena associated with sprites.
\end{abstract}

Keywords. Meteorology and atmospheric dynamics (Lightning; Mesoscale meteorology; Middle atmosphere dynamics)

\section{Introduction}

Transient luminous events (TLEs) that occasionally occur above a large thunderstorm at stratospheric and mesospheric altitudes have been studied intensively during the last two decades and much is now known of their properties. Since their discovery (Franz et al., 1990), several different types of TLEs were well documented in the ground-based observations (e.g. Neubert et al., 2008, and references herein) as well as in aircraft (Sentman and Wescott, 1993; Sentman et al., 1995; Wescott et al., 1995), satellite (Chern et al., 2003; Mende et al., 2005; Cummer et al., 2006a; Chen et al., 2008), and space shuttle measurements (Boeck et al., 1992; Yair et al., 2004).

The TLEs can be divided into several classes depending on their properties (e.g. see Chen et al., 2008). Conventionally, these are sprites/red sprites, blue jets, elves and recently discovered blue starters and gigantic jets. With a luminous red glow occurring at 50-90 km altitude range gradually changing to blue color below $50 \mathrm{~km}$, sprites are more frequent than others in ground-based observations (Sentman et al., 1995; Pasko, 2006). Almost without exceptions, the sprites are associated with positive cloud-to-ground (CG) lightning discharges lowering positive charges from clouds to the ground (Boccippio et al., 1995; Williams et al., 2007). Some events of sprites triggered by negative CGs have been observed, and thus these observations are of great interest (e.g. see Taylor et al., 2008). A sprite can expand upward and downward from the initiation point with velocity of the order of $10^{4} \mathrm{~km} \mathrm{~s}^{-1}$ (e.g. Stanley et al., 1999; Cummer et al., 2006b). 
Their visible sprite duration is from a few to several tens of ms. Typically, the sprite consists of the upper diffuse region in red color and lower tendril-like filamentary structure in blue color with lateral dimension from $20-30 \mathrm{~km}$ to 50 100 km (e.g. Pasko, 2006; Neubert et al., 2008; StenbaekNielsen and McHarg, 2008; Montanyá et al., 2010).

A visible inner structure of the tendrils and branches is very complicated due to presence of bright streamer heads moving upward and downward at velocity $\sim 10^{4} \mathrm{~km} \mathrm{~s}^{-1}$ (Stenbaek-Nielsen et al., 2007). The streamer head scale size is believed to be from $\sim 10 \mathrm{~m}$ to $\sim 100 \mathrm{~m}$.

Blue jets (BJs) are beams of luminosity propagating upwards into the stratosphere from the tops of thunderclouds and exhibiting primarily blue color (e.g. Wescott et al., 1995; Boeck et al., 1995; Mishin and Milikh, 2008). BJs propagate with velocity of the order of $100 \mathrm{~km} \mathrm{~s}^{-1}$ up to $40-50 \mathrm{~km}$ altitude which gives a jet lifetime of $300 \mathrm{~ms}$. It appears that the blue jet initiation results from charge buildup at the top of a thundercloud, and it may or may not be associated with CG flashes (e.g. Neubert et al., 2008). Blue starters differ from BJs jets by a lower terminal altitude since they stop at maximum altitude about $25 \mathrm{~km}$ (Wescott et al., 1996; Heavner et al., 2000; Pasko, 2006), whereas another type of jets termed gigantic jets (GJs) (Wescott et al., 2001; Pasko et al., 2002; Su et al., 2003) has a much greater length and can propagate up to the bottom of the ionosphere.

The GJs occasionally occurring between thundercloud top and lower ionosphere manifest themselves as more intensive discharges than the BJs do. Their upward propagation results in formation of electrical connection between thundercloud tops and the conducting E-layer of the ionosphere (Wescott et al., 2001; Pasko et al., 2002; Su et al., 2003; van der Velde et al., 2007; Kuo et al., 2009; Cummer et al., 2009). Groundbased photographic and video-recordings of TLEs reveal a filamentary structure of the BJs and GJs.

Excitation of molecules of $\mathrm{N}_{2}$ and $\mathrm{O}_{2}$ by electron impact is assumed to be the main cause of red and blue optical emissions associated with TLEs. Above $50 \mathrm{~km}$, the emissions of the first positive band of $\mathrm{N}_{2}\left(\mathrm{~N}_{2} 1 \mathrm{P}\right)$ prevail over other emissions that result in the predominance of red color in the optical emission. At altitudes below $50 \mathrm{~km}$, the strong quenching of $B^{3} \Pi_{\mathrm{g}}$ state gives rise to the suppression of $\mathrm{N}_{2} 1 \mathrm{P}$ emission (e.g. see Vallance-Jones, 1974). The result is that below $50 \mathrm{~km}$ the blue optical emissions become dominant mainly due to excitation of the second positive band of $\mathrm{N}_{2}\left(\mathrm{~N}_{2} 2 \mathrm{P}\right)$. We cannot come close to exploring these topics in any detail since in this paper the main emphasis is on major generation mechanisms for TLEs. The reader is referred to the reviews by Pasko $(2006,2010)$ for the details on optical emissions associated with TLEs.

An "elf" is a concentric ring of optical emissions associated with a CG flash of either polarity. The elves, which are visible for less than $0.1 \mathrm{~ms}$, can appear at the bottom of ionosphere at $\sim 90 \mathrm{~km}$ altitude and then expand over $300 \mathrm{~km}$ laterally (Boeck et al., 1992; Fukunishi et al., 1996; Inan et al., 1996a, b, 1997; Cheng et al., 2007). The elves got their name as an abbreviation for Emission of Light and VLF perturbations due to EMP Sources (Fukunishi et al., 1996). They are caused by the perturbation of ionospheric plasma due to a strong electromagnetic impulse radiated by the CG discharge current (e.g. see Nickolaenko and Hayakawa, 1995; Inan et al., 1996b; Cho and Rycroft, 1998; Rowland, 1998; Kuo et al., 2007). In this review, we do not concern a theory of this effect and refer the reader to a discussion by Rowland (1998) for details.

By convention, the theories of lightning-produced phenomena can be split into two groups depending on the mechanism of air breakdown. The first group is based on the mechanism of conventional air breakdown, that is, on the thermal ionization of the air by low energy electrons in the presence of electric fields (e.g. see Pasko et al., 1995, 1996a, b, 1997a, b, 1998a; Winckler et al., 1996; Fernsler and Rowland, 1996; Pasko, 2006; Raizer et al., 2007; Krehbiel et al., 2008; Riousset et al., 2010a, b; Raizer et al., 2010). Typical energy of electrons producing the ionization is about 10$20 \mathrm{eV}$ while the mean electron energy is about $2 \mathrm{eV}$. This socalled conventional breakdown occurs as the electric field exceeds the threshold value. The second group of the theories assumes the relativistic runaway electrons as the proper candidate for producing the air breakdown at stratospheric and mesospheric altitudes (e.g. see Gurevich et al., 1992, 1994, 1996, 2000; Roussel-Dupré et al., 1994; Roussel-Dupré and Gurevich, 1996; Lehtinen et al., 1997, 1999; Babich et al., 1998, 2008; Gurevich and Zybin, 1998, 2001; Lehtinen, 2000; Füllekrug et al., 2010, 2011). The runaway breakdown requires the presence of seed relativistic electrons with energies greater than $1 \mathrm{MeV}$.

To treat the electromagnetic phenomena in plasma associated with TLEs, a set of transport kinetic and Maxwell's equations is required, which, in their full form, are rather complicated. Thus, the most portion of theoretical study is based on numerical calculations and only a few papers include simple estimates of the TLEs parameters. It is not surprising, then, that the interpretation of the recent observations is basically based on results of numerical modeling of the TLEs (e.g. see special issue J. Phys. D Appl. Phys., 41, 2008; recent reviews by Ebert and Sentman, 2008; Roussel-Dupré et al., 2008; Pasko, 2010; Ebert et al., 2010; Inan et al., 2010; Milikh and Roussel-Dupré, 2010, and references therein).

The main goal of this review is to present an overview of basic physical principles and mechanisms thought to be a major contributor to the TLEs. The analytical results are of our prime interest here despite that they are not so numerous. In order to gain a better understanding of these mechanisms and to make physics of phenomena as transparent as possible, we chose the simplest way to estimate basic parameters of the TLEs. Here our approach is much more modest in scope, aiming at physical intuition rather than detailed analysis.

After some introductory material, the study is begun with the conventional mechanism for air breakdown at 
stratospheric and mesospheric altitudes. In this section, a streamer size and mobility of electrons as a function of altitude in the atmosphere are estimated on the basis of similarity law. Section 3 covers the basics of runaway electron breakdown. In Sect. 4, we review different streamer-leader models of jet and sprite discharges and examine effect of thunderclouds charge distribution on their initiation. In the remainder of this review, the current problems of TLEs simulation are discussed.

\section{Conventional mechanism for high-altitude air breakdown}

Wilson (1925) was the first to establish the crucial role of thundercloud static electric fields that can exceed the breakdown threshold of air at higher altitude above thunderclouds. Despite that the electrical structure of a typical thundercloud is rather a stratiform, the most part of positive charges tend to pile up at the upper portion of the thundercloud, whereas most of negative charges predominantly accumulate at its bottom (Lyons et al., 2003; Lyons, 2006; Krehbiel et al., 2008; Pasko, 2010). The simplest model of the spatial charge separation in the thundercloud is shown in Fig. 1. The separation of oppositely charged particles is thought to be due to slow hydrodynamics processes inside the thundercloud. It appears that updrafts drag small and light positively charged ice fragments, whereas heavy negatively charged hailstones predominantly fall downward due to gravity. Since the current is upward inside the thundercloud and approximately zero outside, charges pile up at the thundercloud boundaries as shown in Fig. 1. Here $\boldsymbol{j}_{\mathrm{g}}$ is updrafts- and gravitydriven current density inside the thundercloud. This current causes the charge separation in the thundercloud, and thus it plays a role of a battery/source for generation of upward or downward-directed lightning discharges. $\boldsymbol{j}_{\mathrm{f}}$ denotes the socalled fair weather current, that is, a weak current flowing from mesosphere to the ground. The lightning can be operative as long as the current $\boldsymbol{j}_{\mathrm{g}}$ can separate the charges and provide the top of the thundercloud with sufficient amount of positive charges.

It is usually the case that the net charge of certain sign amounts to several tens of coulombs, which yields a strong quasi-electrostatic (QE) electric field inside and around the thundercloud. Certainly, this is only an idealized geometry of the thundercloud that ignores actual multi-layered charge distribution inside the thundercloud. We return to this point in more detail in Sect. 4.1. A model of BJ propagation shown in the top of Fig. 1 is discussed in Sect. 4.2.

In this section, we first study a conventional thermal breakdown of air in the presence of a strong QE field, which occasionally occurs inside and around thunderclouds.

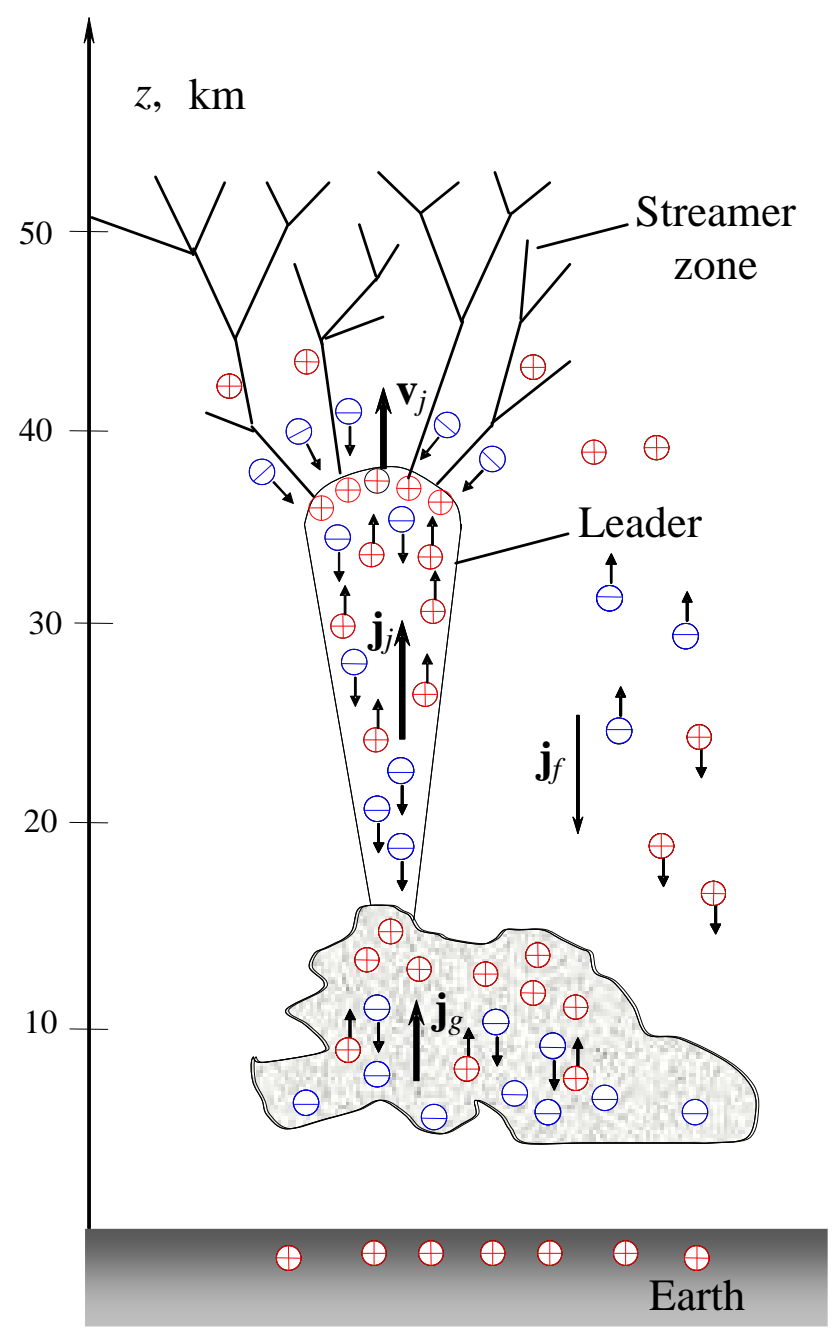

Fig. 1. The simplest model of spatial charge distribution in thundercloud and a scenario of blue jet development considered as a gigantic leader-like discharge. This figure is partly adapted from Uman (1987) and Pasko et al. (1996a). Here $\boldsymbol{v}_{\mathrm{j}}$ is the vector of blue jet velocity, $j_{\mathrm{g}}$ is updrafts- and gravity-driven current density, while $\boldsymbol{j}_{\mathrm{j}}$ and $\boldsymbol{j}_{\mathrm{f}}$ are the conduction current density and fair weather mean current density, respectively.

\subsection{Ionization and electron attachment to neutrals}

The near-surface air conductivity is mainly due to the presence of positive and negative ions, charged aerosols and dust particles, whereas the concentration of free electrons is very small. The air breakdown followed by the production of free electrons results in enhancement of the electron contribution to the air conductivity. It is customary to assume that the free electrons can play a crucial role in the ionization of neutrals in the ambient electric field, because their mobility is much greater than that of ions.

A conventional CG lightning originates from propagation of negative/positive leaders that are in turn surrounded by streamer zones. The streamer zone of leader contains a highly 
branched system of the streamer channels (Uman, 1987; Rakov and Uman, 2003). The conductivity at streamer head is mainly controlled by the dominant inelastic processes for energized electrons such as the ionization and electronic excitation of neutrals and electron attachment to neutrals.

The changes in electron number density $N_{\mathrm{e}}$ are described by the following equation (e.g. see Ebert et al., 2006):

$\partial_{\mathrm{t}} N_{\mathrm{e}}+\nabla \cdot\left(\boldsymbol{v}_{\mathrm{d}} N_{\mathrm{e}}\right)=\left(v_{\mathrm{i}}-v_{\mathrm{a}}\right) N_{\mathrm{e}}+\nabla \cdot\left(D_{\mathrm{e}} \nabla N_{\mathrm{e}}\right)+S_{\mathrm{ph}}$,

where $\boldsymbol{v}_{\mathrm{d}}$ is the drift velocity of electron due to electromagnetic field, $v_{\mathrm{i}}$ and $v_{\mathrm{a}}$ are the ionization and dissociative attachment rates, correspondingly, $D_{\mathrm{e}}$ is the coefficient of electron diffusion and the term $S_{\mathrm{ph}}$ stands for the nonlocal photoionization. All the transport and ionization parameters depend on altitude.

To simulate electrodynamics of the sprites and BJs, it is necessary at this point to construct a suitably idealized model of the medium. For simplicity, the effects of electron-ion pair production due to photoionization and electron-ion recombination are assumed to be negligible (e.g. see Pasko, 2006; Asano et al., 2009a, b). According to the early theory of thermal breakdown, the electron density variation due to electron mobility and diffusion inside the jet are neglected as well, so that the changes in electron density are calculated by taking into account only the effects of electron impact ionization and dissociative attachment, which is described by the first term on the right-hand side of Eq. (1) (Pasko et al., 1997b; Veronis et al., 1999). The ionization rate $v_{i}$ depends on the density of neutrals, and on the applied electric field through electron temperature. As $v_{\mathrm{i}}<v_{\mathrm{a}}$, the electron number density falls off quickly due to the dissociative attachment of electrons to electronegative species, essentially to molecules of $\mathrm{O}_{2}$. In the inverse case, i.e. $v_{\mathrm{i}}>v_{\mathrm{a}}$, the electron density may increase exponentially with time creating electron avalanches. The critical value of electric field at which $v_{\mathrm{i}}=v_{\mathrm{a}}$ is known as the conventional breakdown electric field, $E_{k}$.

In the air, the impact ionization of oxygen and nitrogen molecules plays a major role in production of the free electrons:

$$
\begin{aligned}
& e^{-}+\mathrm{O}_{2} \rightarrow 2 e^{-}+\mathrm{O}_{2}^{+}, \\
& e^{-}+\mathrm{N}_{2} \rightarrow 2 e^{-}+\mathrm{N}_{2}^{+} .
\end{aligned}
$$

The dissociative attachment of electrons to molecules of $\mathrm{O}_{2}$ prevails as the energetic electrons overcome the $3.6 \mathrm{eV}$ energy barrier:

$\mathrm{O}_{2}+e^{-} \rightarrow \mathrm{O}^{-}+\mathrm{O}$.

The rate $v_{\mathrm{a}}$ of this reaction is dependent on the applied electric field through electron temperature and is proportional to the neutral molecule number density $N_{\mathrm{m}}$, which falls off approximately exponentially with altitude $z$ :

$N_{\mathrm{m}} \approx N_{0} \exp (-z / h)$, where $h \approx 8-10 \mathrm{~km}$ and denotes the height of standard atmosphere. According to Barrington-Leigh (2000), the rate $v_{\mathrm{a}}$ tends to maximize at an electric field value slightly below the conventional breakdown threshold $E_{k}$, and the maximum reaction rate can be roughly approximated by the following: $\log v_{\mathrm{a}}=7.9-0.065 z$, where $v_{\mathrm{a}}$ is measured in $\mathrm{s}^{-1}$ and $z$ in $\mathrm{km}$.

The proper conditions for the reaction (3) may exist in the upper atmosphere. For example, consider a BJ emanating from the top of a thundercloud and propagating up to the stratospheric altitude $(\sim 50 \mathrm{~km})$ with the mean velocity, $v_{j}$, about $100 \mathrm{~km} \mathrm{~s}^{-1}$. Although the inverse reaction rate $v_{\mathrm{a}}^{-1}$ varies from $\sim 0.2 \times 10^{-6}$ to $\sim 10^{-5} \mathrm{~s}$ (nighttime conditions) in the $20-50 \mathrm{~km}$ altitude range, it is still much smaller than the typical time $(\sim 0.3 \mathrm{~s})$ of $\mathrm{BJ}$ propagation. Moreover, this means that the energetic electrons at the jet front may disappear for a short distance $\sim v_{j} v_{\mathrm{a}}^{-1} \approx 0.02-1 \mathrm{~m}$ due to the dissociative attachment to $\mathrm{O}_{2}$. This reaction seems to be more important at the mesospheric altitude typical for the sprites.

A plausible candidate, which is capable of explaining the persistence of electron density behind the jet front for a long time, is the three-body attachment processes involving either $\mathrm{O}_{2}$ and $\mathrm{N}_{2}$ or two $\mathrm{O}_{2}$ molecules (Glukhov et al., 1992):

$\mathrm{O}_{2}+e^{-}+A \rightarrow \mathrm{O}_{2}^{-}+A+0.5 \mathrm{eV}$,

where $A$ is another neutral molecule. The rate $v_{\mathrm{a}}$ of this reaction is a function of the electric field as well and is approximately proportional to the neutral molecule number density $N_{\mathrm{m}}$ squared. In this case, the relationship between the peak value of $v_{\mathrm{a}}$ and $z$ can be roughly approximated by the following: $\log v_{\mathrm{a}}=7.9-0.13 z$ (Barrington-Leigh, 2000), where $v_{\mathrm{a}}$ is measured in $\mathrm{s}^{-1}$ and $z$ in $\mathrm{km}$. In the same altitude range, the nighttime inverse rate $v_{\mathrm{a}}^{-1}$ varies from $\sim 0.2 \times 10^{-5}$ to $\sim 2 \times 10^{-1}$ s. It is important that first the rate of the threebody reaction given by Eq. (5) is slower than the rate of reaction given by Eq. (3), and secondly the energy barrier for the reaction (5) is absent. The interested reader is refereed to the special issue on streamer, sprites and lightning (J. Phys. D. Appl. Phys., 2008, 41, No. 23 and references herein) for a more complete treatise on the role played by both attachment reactions in TLEs generation.

Laboratory tests of low-pressure electric discharges in air have shown that the electron attachment can be accompanied by inverse fast process of associative attachment (Moruzzi and Price, 1974; Rayment and Moruzzi, 1978). This process enhances at the pressure below $0.1 \mathrm{~atm}$, which corresponds to the altitudes above $15 \mathrm{~km}$. Recently, Luque and Gordillo-Vázquez (2011) have assumed that the electron realized through associative detachment to $\mathrm{N}_{2}$,

$\mathrm{O}^{-}+\mathrm{N}_{2} \rightarrow \mathrm{N}_{2} \mathrm{O}+e^{-}$,

can be of great importance for the sprite generation. According to Neubert et al. (2011), the detachment rate can be greater than both the ionization and attachment rates only if 
the normalized electric field at $70 \mathrm{~km}$ altitude $E / E_{k}<0.38$, where $E_{k}$ denotes the breakdown threshold, while the dissociative attachment prevails over detachment and ionization as $0.38<E / E_{k}<1$ (e.g. see Fig. 4 in the paper by Neubert et al., 2011). As compared to the above processes, the threebody attachment given in Eq. (5) is thought to be of minor importance above $40 \mathrm{~km}$ altitude.

\subsection{Conventional breakdown threshold}

Laboratory experiments have shown that the main characteristics of gas discharge depend on the gas pressure $p$ and inter-electrode gap size $d$ in such a way that the breakdown voltage $V_{\mathrm{b}}$ is approximately proportional to $p d$ at least under condition $p d>10^{6} \mathrm{~Pa}$ m (e.g. see Raizer, 1991; Lieberman and Lichtenberg, 1994). The breakdown threshold is proportional to the gas pressure because $E_{k} \approx V_{\mathrm{b}} / d \sim p$. We have $p=N_{\mathrm{m}} k_{\mathrm{B}} T$ where $k_{\mathrm{B}}$ stands for Boltzmann constant, so that the value of $E_{k}$ is proportional to number density $N_{\mathrm{m}}$ of the neutral gas at constant gas temperature, $T$. The same tendency is assumed to be valid at the altitudes where BJs, GJs, sprites, and other TLEs occur. For example according to $\mathrm{Pa}-$ padopoulos et al. (1993), the conventional breakdown threshold in the air is related to the ambient gas number density through

$E_{k}=32 N_{\mathrm{m}} / N_{0}, \mathrm{kV} \mathrm{cm}^{-1}$.

Here $N_{0}=2.7 \times 10^{25} \mathrm{~m}^{-3}$ and is the constant of the order of air number density at the ground level. According to Eqs. (4) and (7), the conventional breakdown threshold falls off approximately exponentially with altitude along with the air number density.

Considering the important role played by the plasma conductivity in the electric discharges, we dwell on electron/ion mobility $\mu$, which is defined by $v_{\mathrm{d}}=\mu E$, where $v_{\mathrm{d}}$ is the drift velocity of charged particles. According to the elementary theory, the electron/ion mobility in a weakly ionized gas is given by

$\mu_{\mathrm{e}, \mathrm{i}}=\frac{e}{m v_{\mathrm{en}, \mathrm{in}}}$,

where $v_{\mathrm{en}}$ and $\nu_{\text {in }}$ are the elastic electron-neutral and ionneutral collision frequencies, respectively, $e$ is elementary charge and $m$ denotes the reduced mass of the electron/ion and neutral. Here the electron-ion collisions were ignored. The collision frequency can be roughly estimated through $v=\bar{v} / \lambda \sim \bar{v} \sigma_{\mathrm{c}} N_{\mathrm{m}}$, where $\lambda$ denotes the free length of charged particles, and $\bar{v}$ and $\sigma_{\mathrm{c}}$ are the mean particle velocity and scattering cross section, respectively. It immediately follows from Eq. (8) and above estimates that the mobility of charged particles is inversely proportional to the neutral number density.

The main distinctive property of electron mobility manifests itself through the dependence $\mu_{\mathrm{e}}$ not only on $N_{\mathrm{m}}$ but also on the ratio $E / N_{\mathrm{m}}$ (e.g. see Huxley and Crompton, 1974; Eletsky et al., 1975). The reason is that the electrons have lost a small amount of their kinetic energy by elastic collisions with neutrals since the electron mass is much smaller than the neutral mass. It is usually the case that, even with a weak electric field, the mean electron energy exceeds the mean energy of heavy particles. The mean electron energy $\bar{\varepsilon}$ in the weakly ionized gas is approximately proportional to the energy that the electron would acquire between two successive collisions with neutrals, that is, $\bar{\varepsilon} \propto$ $e E \lambda \sim e E /\left(\sigma_{\mathrm{c}} N_{\mathrm{m}}\right)$, where the scattering cross section $\sigma_{\mathrm{c}}(\bar{\varepsilon})$ is a complex function of $\bar{\varepsilon}$. This relationship is an implicit function which can be solved for $\bar{\varepsilon}$ or for $\bar{v}$ as a function of $E / N_{\mathrm{m}}$. This means that both the collision frequency and electron mobility are dependent on $E / N_{\mathrm{m}}$ as well.

However, the experimental study of the dependence $\mu_{\mathrm{e}}\left(E / N_{\mathrm{m}}\right)$ shows that the electron mobility is almost constant as a function of $E / N_{\mathrm{m}}$ within an interval from $10^{-21}$ to $10^{-19} \mathrm{~V} \mathrm{~m}^{2}$ for a variety of gases including $\mathrm{N}_{2}, \mathrm{CO}_{2}$, etc. (Eletsky et al., 1975). On account of the estimate $E_{k} / N_{\mathrm{m}} \approx$ $1.2 \times 10^{-19} \mathrm{~V} \mathrm{~m}^{2}$ that follows from Eq. (7), one may expect that the electron mobility is only dependent on $N_{\mathrm{m}}$ as the electric field is close to the conventional breakdown threshold. For example, Pasko et al. (1996a) have used the relationship

$\mu_{\mathrm{e}} \approx 4 \times 10^{-2} N_{0} / N_{\mathrm{m}},\left(\right.$ in $\left.\mathrm{m}^{2}(\mathrm{~V} \mathrm{~s})^{-1}\right)$

for $E \approx E_{k}$ to interpret the results of their numerical simulation of blue jets.

The numerical value of the electron drift velocity for $E \approx$ $E_{k}$ can be estimated through $v_{\mathrm{d}} \sim \mu_{\mathrm{e}} E_{k}$. Substituting Eq. (7) for $E_{k}$ and Eq. (9) for $\mu_{\mathrm{e}}$ into the above equation for $v_{\mathrm{d}}$ yields $v_{\mathrm{d}} \approx 130 \mathrm{~km} \mathrm{~s}^{-1}$. Notice that this value is of the order of the BJ velocity $v_{j}=40-130 \mathrm{~km} \mathrm{~s}^{-1}$ (e.g. see Neubert et al., 2008).

Returning to the reaction rates, we note that both values (i.e. $v_{\mathrm{i}}$ and $v_{\mathrm{a}}$ ) are dependent on the electric field and electron mobility (Eq. 9). The impact ionization rate deduced from Townsend approximation is frequently used for the streamer simulations (e.g. see Ebert et al., 2006):

$v_{\mathrm{i}}=\mu_{\mathrm{e}} \alpha_{\mathrm{i}} E \exp \left(-E_{\mathrm{i}} / E\right)$.

On account of the scaling factor $N_{0} / N_{\mathrm{m}}$, the Townsend ionization rate and ionization field are given by $\alpha_{\mathrm{i}}=\alpha_{\mathrm{i} 0} N_{\mathrm{m}} / N_{0}$ and $E_{\mathrm{i}}=E_{\mathrm{i} 0} N_{\mathrm{m}} / N_{0}$. The similar approximation of the electron attachment rate has been recently used by Luque and Ebert (2009) to describe the sprite streamer development:

$v_{\mathrm{a}}=\mu_{\mathrm{e}} \alpha_{\mathrm{a}} E \exp \left(-E_{\mathrm{a}} / E\right)$,

where the Townsend attachment rate and attachment field are given by $\alpha_{\mathrm{a}}=\alpha_{\mathrm{a} 0} N_{\mathrm{m}} / N_{0}$ and $E_{\mathrm{a}}=E_{\mathrm{a} 0} N_{\mathrm{m}} / N_{0}$. 


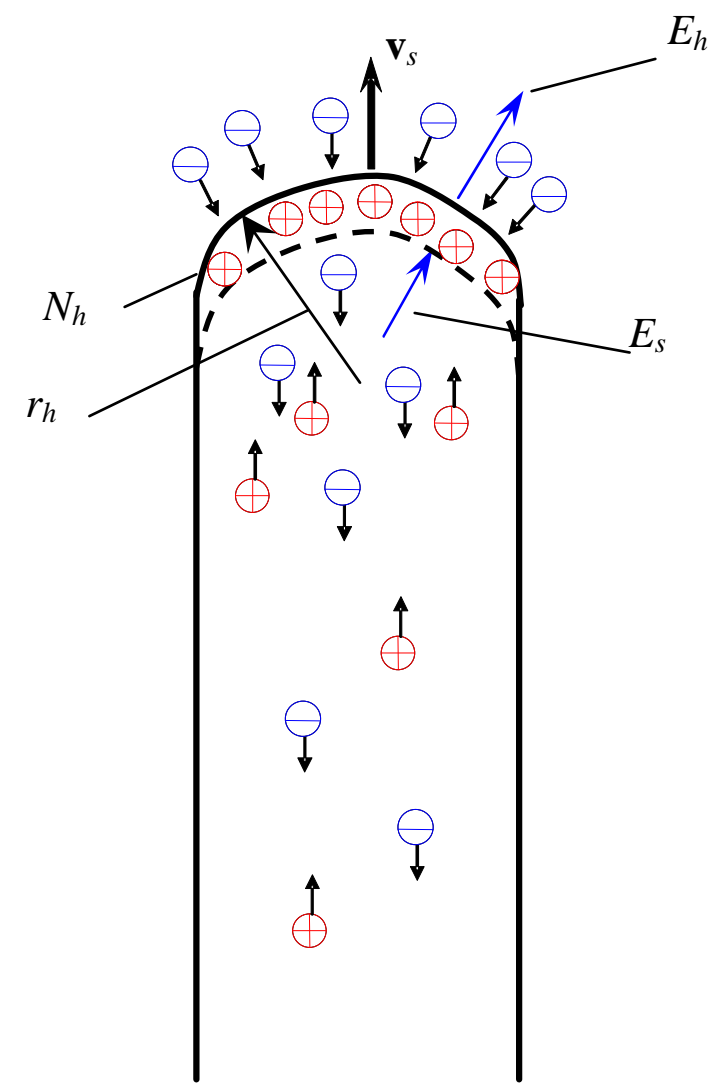

Fig. 2. A positive streamer model. Here $v_{\mathrm{S}}$ is streamer velocity, $r_{\mathrm{h}}$ is curvature radius of the streamer head, $N_{\mathrm{h}}$ is number density of uncompensated/unbalanced positive charges, $E_{\mathrm{h}}$ is electric field in front of the streamer head, and $E_{\mathrm{S}}$ is electric field inside the streamer.

The breakdown threshold is derivable from the condition $v_{\mathrm{i}}=v_{\mathrm{a}}$ and Eqs. (10) and (11):

$E_{k}=\frac{N_{\mathrm{m}}\left(E_{\mathrm{i} 0}-E_{\mathrm{a} 0}\right)}{N_{0} \ln \left(\alpha_{\mathrm{i} 0} / \alpha_{\mathrm{a} 0}\right)}$.

Substituting numerical parameters $E_{\mathrm{i} 0}=2 \times 10^{2} \mathrm{kV} \mathrm{cm}^{-1}$, $E_{\mathrm{a} 0}=30 \mathrm{kV} \mathrm{cm}^{-1}, \quad \alpha_{\mathrm{i} 0}=4.3 \times 10^{3} \mathrm{~cm}^{-1} \quad$ and $\quad \alpha_{\mathrm{a} 0}=$ $20 \mathrm{~cm}^{-1}$ (Luque and Ebert, 2009), we obtain that $E_{k} \approx 32 N_{\mathrm{m}} / N_{0} \mathrm{kV} \mathrm{cm}^{-1}$, which is compatible with Eq. (7).

\subsection{Streamers}

As the electric field becomes much greater than the breakdown threshold (Eq. 7), air breakdown may take place instead in the form of streamers (Bazelyan and Raizer, 1998), which are often referred to as ionization waves. A typical streamer is the self-propagating narrow filamentary of cold low-conducting plasma which can propagate at the velocity $10^{2}-10^{4} \mathrm{~km} \mathrm{~s}^{-1}$ as measured at the ground pressure. Once the steamer is generated, it can propagate through the region where the electric field is smaller than $E_{k}$. Initiation of streamers due to individual electron avalanches will occur most likely in the vicinity of the sharp portion of an electrode, which is typical for point-to-plane corona discharges (e.g. Raizer et al., 1998; Pasko, 2006). The charge density at streamer head is so large that the electric field in the vicinity of the head can reach a value about 4-7 times $E_{k}$ (Celestin and Pasko, 2010). The electron impact and photo-ionization in the streamer head cause an enhancement of ionization coefficient up to the value occurring at the streamer channel that results in moving forward of the streamer channel.

There are two types of streamers, i.e. positive and negative, depending of the sign of the space charge in their heads. A negative streamer propagates due to ejection of electrons from its head. By contrast, the head of a positive streamer propagates due to injections of ambient/seed electron avalanches from surroundings. The strong electric field caused by the spatial charge of the head results in the high rate of impact and photoionization at the streamer front thereby producing the seed electrons (Bazelyan and Raizer, 1998). In electronegative gases, such as air, the presence of oxygen and nitrogen ions and fast electron detachment in an electric field could be an effective source of seed electrons, which in turn results in the positive streamer head formation and leads to the streamer branching phenomena (Pancheshnyi, 2005). Other channels of the seed electron production are cosmic rays and photo-ionization of air due to solar radiation.

The minimum value of electric field required for the propagation of positive streamers in the air at ground pressure is $E_{\mathrm{cr}}^{+}=4.4 \mathrm{kV} \mathrm{cm}^{-1}$, while the same value for negative streamers is $E_{\mathrm{cr}}^{-}=-12.5 \mathrm{kV} \mathrm{cm}^{-1}$ (Raizer, 1991; Allen and Ghaffar, 1995; Babaeva and Naidis, 1997; Pasko, 2006). It should be emphasized once again that the streamer initiation requires an electric field greater than above critical fields and greater than the convention breakdown threshold given by Eq. (7). The requirements for transition from an electron avalanche to a streamer generation also include the critical number of avalanching electrons and a minimum radius of the avalanche region, sharp points of the electrode surface to enhance the local electric field, and many other factors (e.g. Raizer et al., 1998; Bazelyan and Raizer, 1998). Streamer breakdown is not addressed in any detail in the present work, and the reader is referred to above works for details about streamers.

In what follows, we estimate the streamer parameters based on similarity law and on dimension attributes rather than on detailed analysis. Consider, for example, a positive upward-propagating streamer schematically shown in Fig. 2. Once the seed electrons have moved through the streamer head boundary, the uncompensated/unbalanced positive spatial charges arise on their spot, that is, in the layer around the streamer head. This positively charged layer can spread upward together with the streamer head at the streamer velocity (Raizer et al., 2010). The electric field $E_{\mathrm{h}}$ of the 
uncompensated charge is described by Maxwell's equation:

$\nabla \cdot \boldsymbol{E}=e N_{\mathrm{h}} / \varepsilon_{0}$,

where the number density, $N_{\mathrm{h}}$, of the uncompensated positive charges is of the order of the number density, $N_{\mathrm{e}}$, of seed electrons flowing through the head boundary. Let $r_{\mathrm{h}}$ be curvature radius of the streamer head. Substituting the relationship $|\nabla \cdot \boldsymbol{E}| \sim E_{\mathrm{h}} / r_{\mathrm{h}}$ into Eq. (13), we obtain a rough estimate of the electric field in front of the streamer head: $E_{\mathrm{h}} \approx e N_{\mathrm{e}} r_{\mathrm{h}} / \varepsilon_{0}$.

Now we shall show that the field $E_{\mathrm{h}}$ is greater than the electric field $E_{\mathrm{S}}$ inside the streamer body. The surface charge density at the head boundary can be estimated as $q /\left(\pi r_{\mathrm{h}}^{2}\right)$, where $q$ is the total positive charge at the streamer head. Assuming for the moment that the boundary/front of the streamer head is very thin, then the electric field component normal to the boundary has a jump (Fig. 2):

$E_{\mathrm{h}}-E_{\mathrm{s}} \approx q /\left(\pi \varepsilon_{0} r_{\mathrm{h}}^{2}\right)$.

The current density of the seed electrons flowing into the streamer head from the surroundings can be estimated then as follows: $j_{\mathrm{h}} \sim e N_{\mathrm{e}} v_{\mathrm{s}}$, where $v_{\mathrm{S}}$ stands for the streamer front velocity. Normal component of the electric current density must be continuous at the boundary of streamer head, so that $e N_{\mathrm{e}} v_{\mathrm{s}} \sim \sigma_{\mathrm{s}} E_{\mathrm{s}}$, where $\sigma_{\mathrm{s}}$ stands for the conductivity inside the streamer.

Combining the above estimates, we come to

$\frac{E_{\mathrm{s}}}{E_{\mathrm{h}}} \approx \frac{v_{\mathrm{s}} \varepsilon_{0}}{r_{\mathrm{h}} \sigma_{\mathrm{s}}}$

which coincides with estimate given by Vitello et al. (1994) and by Pasko et al. (1996a). Substituting numerical parameters into Eq. (15) shows that the value $E_{\mathrm{s}} / E_{\mathrm{h}}$ can vary in the range $0.1<E_{\mathrm{s}} / E_{\mathrm{h}}<1$ (Pasko et al., 1996a). Thus, to the first approximation, the field $E_{\mathrm{S}}$ in Eq. (14) can be considered as negligible compared with $E_{\mathrm{h}}$. This is not a surprise given the strong electric field produced by a great number of charges situated around the head of streamer.

A streamer-like structure of BJs, GJs and sprites has been well documented with a high-speed camera and with a highresolution detector in many observations (Wescott et al., 2001; Pasko and George, 2002; Cummer et al., 2006a, b; Pasko, 2007; McHarg et al., 2007). As we have noted above, the ionizing collisions and photoionization are predominant in the vicinity of streamer head because of stronger electric field, which in turn causes the charge buildup at the head of streamer (Fig. 1a). The streamer length is substantially limited by timescale of electron loss due to attachment and recombination. As we have stated above, the typical timescale of the three-body electron attachment is $\tau_{\mathrm{a}} \sim v_{\mathrm{a}}^{-1} \sim N_{\mathrm{m}}^{-2}$. Taking into account that $\tau_{\mathrm{a}} \sim 0.1 \mu \mathrm{s}$ at the ground level, we obtain $\tau_{\mathrm{a}} \sim 10 \mu$ s at the $18 \mathrm{~km}$ altitude, which is typical altitude for BJ nucleation (Pasko, 2010). In this notation, one may expect that the characteristic length of individual streamer, $l_{\mathrm{s}} \sim v_{\mathrm{s}} \tau_{\mathrm{a}}$ ( $v_{\mathrm{s}}$ is the streamer velocity), cannot be large due to such a small value of $\tau_{\mathrm{a}}$ (Raizer et al., 2006, 2007). For example, a typical length of the streamer filaments propagating inside the sprite is of the order of several meters.

A total current $I_{\mathrm{s}}$ flowing through the streamer cross section can be estimated as follows:

$I_{\mathrm{s}} \approx e N_{\mathrm{e}} \mathrm{v}_{\mathrm{d}} \pi r_{\mathrm{h}}^{2}$.

In a steady-state injection of the seed electrons, the total current (Eq. 16) has to be conserved along the streamer tube whereas the electron number density $N_{\mathrm{e}}$ and the head radius $r_{\mathrm{s}}$ can be the functions of streamer length. As the total current is a constant, the charge $q$ at the head of streamer has to be increased in time. One may expect that $q \propto t$ and thus $q$ is directly proportional to the streamer length or the head radius $r_{\mathrm{h}}$. This implies that the head radius $r_{\mathrm{h}}$ in Eq. (14) varies in inverse proportion to the electric field jump $E_{\mathrm{h}}-E_{\mathrm{S}} \sim E_{\mathrm{h}}$ which in turn is of the order of the conventional breakdown threshold $E_{k}$ (Eq. 7); whence it follows that

$r_{\mathrm{h}} \propto N_{\mathrm{m}}^{-1} \propto \exp \{z / h\}$.

As is seen from Eq. (17), the radius and characteristic cross section of the streamer increase with height $z$, and thus they can be much greater than that at ground level pressure. This conclusion is consistent with the observations of sprites and BJs.

Notice that we have just derived the so-called similarity relations for gas discharge according to which typical discharge size $L$ (such as discharge tube length, streamer radius etc.) scales as $L \propto N_{\mathrm{m}}^{-1}$. In a similar fashion, we may obtain that the typical time interval $T$ (such as relaxation time, mean free time and etc.) scales as $T \propto N_{\mathrm{m}}^{-1}$. Typical velocity does not scale, which is independent of $N_{\mathrm{m}}$. The scaling/critical electric field $E \propto N_{\mathrm{m}}$, whereas a typical charge $q \propto N_{\mathrm{m}}^{-1}$.

According to our estimate obtained in Sect. 2.2, the electron drift velocity $v_{\mathrm{d}}$ is approximately constant at the streamer head. Taking the notice of this fact and combining Eqs. (16) and (17), we get $N_{\mathrm{e}}(z) \propto N_{\mathrm{m}}^{2}$. In the same approximation, the streamer conductivity inside the streamer head $\sigma_{\mathrm{s}}=e N_{\mathrm{e}} \mu_{\mathrm{e}} \propto N_{\mathrm{m}}$. By contrast with our estimates, the early numerical modeling of blue jets as streamers (e.g. Pasko et al., 1996a) suggested that $\sigma_{j}$ behind the jet front remains approximately constant. This may be due to the simplified approach, which leaves out of account altitude dependence of ionization and attachment parameters in Eq. (1).

A detailed description of the similarity relations is found in many papers (e.g. see Raizer et al., 1998; Pasko, 2006). From the above analysis, it is clear that the streamer parameters in the sprites and GJs are scaled by reduced air density in the same manner.

Interestingly enough, the recent numerical simulation of sprite behavior has shown that initial stage of sprite formation departs from simple similarity law discussed above 


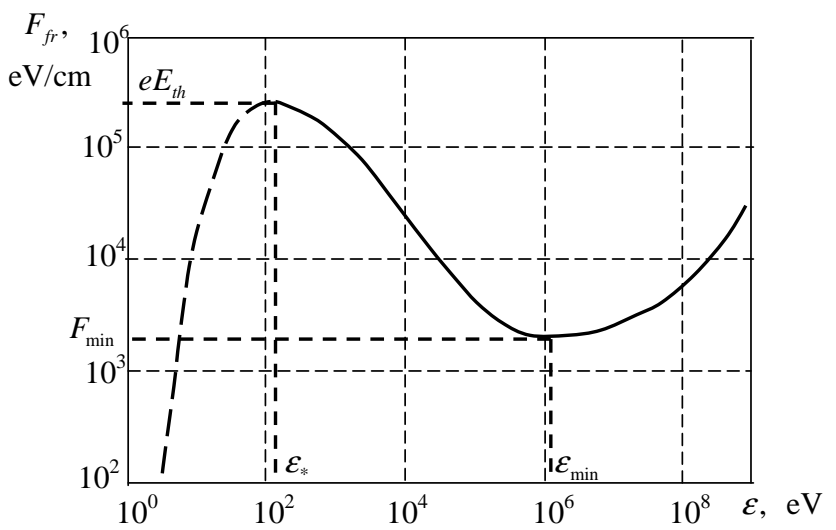

Fig. 3. A schematic plot of dynamical friction force of electrons in the air versus electron kinetic energy. The figure is partly adopted from Pasko (2006).

(Luque and Ebert, 2009, 2010). Moreover, the blue jet velocity is not as constant as the similarity law predicts. Typically, the blue jet is started at the velocity $\sim 40 \mathrm{~km} \mathrm{~s}^{-1}$ and then reaches the value $v_{j}=100-130 \mathrm{~km} \mathrm{~s}^{-1}$ at the altitude range 35-50 km until the jet stops (Pasko et al., 1996a).

To summarize, we note that the theory of streamers/leaders propagating at stratospheric and mesospheric altitudes is still far from being accurate. So, in what follows, our study predominantly focuses on the similarity and dimension law as well as on the scaling properties of the streamers rather than detailed analysis.

\section{Runaway electron breakdown}

When the electric field is greater than the conventional breakdown threshold, it produces the mean thermal electron energy of the order of several $\mathrm{eV}$. In contrast to the thermal breakdown of air, the runaway breakdown is based on the mechanism of relativistic electrons avalanches. One of the merits of this mechanism is that the electric field threshold required for air breakdown may be one order of magnitude lower than that due to the conventional breakdown. In what follows, the runaway electron breakdown will be treated in a sketchy fashion and then this mechanism of air breakdown is examined as a possible source for TLEs.

\subsection{Elementary model of relativistic runaway}

We deal first with the simple one-dimensional model in which all the electrons move along $\mathrm{z}$-axis in opposition to the vector of constant electric field, $\boldsymbol{E}$. To justify this model for electron motion, one should note that the electron forward scattering at small angles prevails if the electron energy is greater than $50 \mathrm{eV}$ (Pasko, 2006). In such a case, the equation of electron motion is given by (Gurevich et al., 1992, 1994) $\frac{d p}{d t}=e E-F_{\mathrm{fr}}(\varepsilon)$

where the so-called dynamical friction force $F_{\text {fr }}(\varepsilon)$, pointed oppositely to the vector of electron momentum $\mathbf{p}$, describes the electron collisions. In a more accurate model, one should take into account the angle included between the electric force $e E$ and the electron momentum (Gurevich et al., 1992; Babich, 2003).

The dynamical friction force equals the electron energy loss due to the electron collisions per unit length:

$F_{\text {fr }}(\varepsilon)=\frac{d \varepsilon}{d z}$.

For high-energy electrons, the friction force is determined predominantly by electron energy losses due to the ionization of air. Since the energy of the fast-moving electron is much greater than the energies of atomic electrons, it interacts with atomic electrons and nuclei as with free particles. A rough estimate of the friction force is $F_{\mathrm{fr}}(\varepsilon) \sim \varepsilon / \lambda$, where the free length of the electrons $\lambda \sim\left(Z N_{\mathrm{m}} \sigma_{\mathrm{c}}\right)^{-1}$ depends on the number density of molecules $N_{\mathrm{m}}$, the mean number of electrons in molecule $Z$, and the scattering cross section $\sigma_{\mathrm{c}}$. In the non-relativistic energy range, the interaction is governed by the Coulomb law through Rutherford scattering cross section $\sigma_{\mathrm{c}} \sim e^{4} / \varepsilon^{2}$ (e.g. see Gurevich and Zybin, 2001). Combining the above relationships, we arrive at the estimate $F_{\mathrm{fr}}(\varepsilon) \sim e^{4} Z N_{\mathrm{m}} / \varepsilon$, which is in good agreement with the formula

$F_{\mathrm{fr}}(\varepsilon)=\frac{2 \pi e^{4} Z N_{\mathrm{m}}}{\varepsilon} \ln \frac{\varepsilon}{J_{z}}$,

derived by Bethe (1930) in a more accurate model. Here $J_{Z} \sim$ $Z \varepsilon_{\mathrm{i}}$, where $\varepsilon_{\mathrm{i}}$ is the energy of ionization.

The dynamical friction force of electrons in air is schematically shown in Fig. 3. As is seen from this figure, at first the friction force approximately increases with the electron kinetic energy up to the energy $\sim 150 \mathrm{eV}$ as shown with dashed line. The maximum of the friction force at the energy $\varepsilon_{*}$ corresponds to the so-called thermal runaway threshold, which occurs at the electric field $E_{\mathrm{th}} \approx 260 \mathrm{kV} \mathrm{cm}^{-1}$. In the $10^{2}-$ $10^{6} \mathrm{eV}$ energy range, the friction force falls off with increasing of the electron energy according to Eq. (20). We recall that this tendency is due to Coulomb interaction of the fastmoving electron with atomic electrons that results in a decrease in scattering cross section.

At higher energies, the contribution of relativistic effects, which we have just ignored, results in gradual changes in the above tendency so that the friction force has a minimum $F_{\min }$ at the energy $\varepsilon_{\min } \approx 1.4 \mathrm{MeV}$, and then a logarithmically slow increase begins due to relativistic effects (Gurevich and Zybin, 2001). 


\subsection{Runaway breakdown}

As illustrated in Fig. 3, the possibility for generating runaway electrons results from the fact that the fall off of the friction force dominates in the energy range from $\varepsilon_{*}$ to $\varepsilon_{\min }$. It follows from Eq. (18) that the runaway electrons can appear under the following requirement:

$e E>F_{\mathrm{fr}}(\varepsilon)$,

which can be valid for the ambient electric field $E>E_{\mathrm{c}}=$ $F_{\min } / e$. The implication here is that, if Eq. (21) holds true, then the electric field will accelerate such electrons continuously so that they become runaway electrons. In the inverse case (that is, if $e E<F_{\text {fr }}(\varepsilon)$ ), the electron energy falls off quickly due to the ionization of air and other inelastic processes resulting in the energy losses.

Taking into account that, in the vicinity of the minimum point, the logarithm in Eq. (20) is approximately equal to $a \approx 11$; the minimal value of the friction force and threshold electric field are given by (Gurevich and Zybin, 2001)

$E_{\mathrm{c}}=\frac{F_{\min }}{e}=\frac{4 \pi e^{3} Z N_{\mathrm{m}} a}{m_{\mathrm{e}} c^{2}}$.

Combining Eqs. (20)-(22), we obtain that the runaway electron energy must be greater than the threshold value, $\varepsilon_{\mathrm{c}}$, which depends on the ambient electric field:

$\varepsilon>\varepsilon_{\mathrm{c}} \approx \frac{m_{\mathrm{e}} c^{2} E_{\mathrm{c}}}{2 E}$.

Generation of large amount of secondary low-energy electrons due to the neutral molecule ionization by runaway electron impact is the main cause of the runaway breakdown of air. Although a majority of secondary electrons have a small energy, a portion of such electrons may have energy greater than the threshold value, that is, $\varepsilon>\varepsilon_{\mathrm{c}}$. The electric field will accelerate these energetic electrons, so that they also become the runaway electrons, which in turn results in additional ionization of air and the generation of a new portion of runaway electrons. As a result, one may expect an exponentially increasing avalanche of runaway electrons (e.g. see Colman et al., 2010). A great deal of the secondary slow electrons is produced along with the runaway electrons, and eventually it brings the breakdown of air.

It should be noted that, if the electric field is greater than $E_{\mathrm{th}} \approx 260 \mathrm{kV} \mathrm{cm}^{-1}$, then the electric force acting on electrons becomes greater than the maximal friction force, which corresponds to the maximum in the graph of Fig. 3. Under such a strong electric field, all the thermal electrons become runaway ones. As is seen from Fig. 3, the thermal runaway breakdown requires the threshold 100 times greater than that given by Eq. (22) (Papadopoulos et al., 1996).

The runaway breakdown field (Eq. 22) is dependent on the neutral number density and thus is dependent on height. On account of Eq. (3), this dependence can be approximated as follows (Gurevich and Zybin, 2001):

$E_{\mathrm{c}}=2.16 \exp (-z / h), \mathrm{kV} \mathrm{cm}^{-1}$,

where $h \approx 8-10 \mathrm{~km}$. It is important that the value of the runaway breakdown threshold $E_{\mathrm{c}}$ is one order of magnitude smaller than the conventional breakdown $E_{k}$. For example, comparing Eq. (24) with Eq. (7), one can find that, at the ground-level pressure, $E_{\mathrm{c}}=2.16 \mathrm{kV} \mathrm{cm}^{-1}$, whereas $E_{k}=32 \mathrm{kV} \mathrm{cm}^{-1}$.

In this section, we have studied the simplified model, in which, as we have noted, a single fast electron is subject to the friction force that approximates the fast electron scattering by electrons and nuclei of molecules. This model ignores the energy losses due to radiations of relativistic electrons, although Roentgen and gamma quanta are able both to ionize the molecules and to generate electron-positron pairs when interacting with nuclei of molecules. In a more accurate theory, the runaway electrons are described by the Boltzmann transport equation for fast electron distribution function $f(\boldsymbol{r}, \boldsymbol{p}, t)$ over the coordinates $\mathbf{r}$ and momenta $\mathbf{p}$ (e.g. see recent reviews by Roussel-Dupré et al., 2008, and by Milikh and Roussel-Dupré, 2010):

$\frac{\partial f}{\partial t}+\boldsymbol{v} \frac{\partial f}{\partial \boldsymbol{r}}+e \boldsymbol{E} \frac{\partial f}{\partial \boldsymbol{p}}=S\left(f, F_{\mathrm{m}}\right)$.

Here $S$ stands for the collision integral dependent on the distribution function of electrons $f$ and neutral molecules $F_{\mathrm{m}}$. Owing to the complexity of Eq. (25) and the collision integral, we omit the analysis of Eq. (25) and its solutions. The interested reader is asked to consult Gurevich and $\mathrm{Zy}$ bin (2001) and Trakhtengertz et al. $(2002,2003)$ for a more complete treatise on runaway electron processes.

\subsection{Characteristic length of avalanche and role played by fast seed electrons}

The energy losses of the fast electrons are mainly due to the ionization of neutrals, which results in the generation of secondary electrons. Let $d N$ be the number of secondary electrons generated over a small length $d z$ due to the ionization of neutrals. In what follows we consider only that part of the secondary electrons that has the energy greater than the certain value $\varepsilon_{\mathrm{s}}$. Additionally, we suppose that the chosen value $\varepsilon_{\mathrm{S}}$ is much smaller than the energy of the incident fast electron. In this case, the number of that part of the secondary electrons $d N\left(\varepsilon_{\mathrm{s}}\right)$ is given by (Landau and Lifshits, 1982)

$\frac{d N\left(\varepsilon_{\mathrm{s}}\right)}{d z}=\frac{\pi Z N_{\mathrm{m}} e^{4}}{m_{\mathrm{e}} c^{2} \varepsilon_{\mathrm{s}}}$.

In the energy range $\varepsilon_{\mathrm{S}}>\varepsilon_{\mathrm{c}}$, the electric field will accelerate the secondary electrons in such a way that they become runaway, giving rise to a runaway electron avalanche. As the 
electric field is parallel to z-axis, the number of runaway electrons increases exponentially with coordinate $z$ :

$N=N_{0} \exp \left(z / l_{\mathrm{a}}\right)$.

The number of slow/thermal electrons increases exponentially along with the runaway electrons.

The rough estimate of the characteristic length $l_{\mathrm{a}}$ of runaway electrons avalanche can be found from Eq. (26) at $\varepsilon_{\mathrm{s}}=\varepsilon_{\mathrm{c}}$ (Gurevich and Zybin, 2001):

$l_{\mathrm{a}}=\left(\frac{d N}{d z}\right)_{\varepsilon_{\mathrm{s}}=\varepsilon_{\mathrm{c}}}^{-1}=\frac{\left(m_{\mathrm{e}} c^{2}\right)^{2}}{2 \pi Z N_{\mathrm{m}} e^{4}} \frac{E_{\mathrm{c}}}{E}$.

Here we have used Eq. (23) for $\varepsilon_{\mathrm{c}}$.

As long as the electric field $\boldsymbol{E}$ is close to the breakdown threshold $E_{\mathrm{c}}$, the characteristic length of exponential growth (Eq. 28) varies approximately inversely proportional to $N_{\mathrm{m}}$. In the atmosphere at the ground surface level, the value of $l_{\mathrm{a}}$ is of the order of several tens of meters. This value increases with altitude due to fall off of the neutral number density $N_{\mathrm{m}}$.

To initiate runaway breakdown, the presence of seed relativistic electrons, which have the energy greater than the critical value, that is $\varepsilon>\varepsilon_{\mathrm{c}}$, is necessary. These seed electrons are produced by cosmic rays and extensive cosmic ray showers. The distribution of primary high-energy cosmic rays with energies above a few $\mathrm{GeV}$ or much greater is well documented over many orders of magnitude in energy (e.g. Nagano and Watson, 2000). The models of runaway breakdown in the atmosphere are based on the assumption that cosmic rays are capable of producing the shower of secondary particles called an extensive air shower (EAS) (Gurevich et al., 1999b; Lehtinen et al., 1999; Gurevich and Zybin, 2001, 2004; Inan and Lehtinen, 2005; Roussel-Dupré et al., 2008; Milikh and Roussel-Dupré, 2010). The incident cosmic ray particle energy to initiate runaway breakdown was estimated to be greater than or of the order of $10^{15} \mathrm{eV}$ (Gurevich et al., 1999b). The flux of the secondary particles typically consists of $89 \%$ photons, $10 \%$ electrons with the energy up to $30 \mathrm{MeV}$ and $1 \%$ other particles, largely muons (e.g. Carlson et al., 2008). For the runaway breakdown, the secondary/seed electrons are the most important.

Since the primary cosmic rays are highly relativistic, the secondary particles all travel predominantly along the primary's direction as a consequence of the principle of conversation of momentum. However, the particles in the electromagnetic cascade of an EAS form a pancake-like structure due to lateral expansion of the EAS caused by the decay of neutral pions into two momentum-conserving gamma rays (Gurevich and Zybin, 2005; Gurevich et al., 2009; Milikh and Roussel-Dupré, 2010). Typically, an EAS has just a few meters along the direction of the primary cosmic ray's motion, but about $100-150 \mathrm{~m}$ across.

It follows from the theory that the EAS-produced currents of relativistic runaway and thermal electrons in the strong

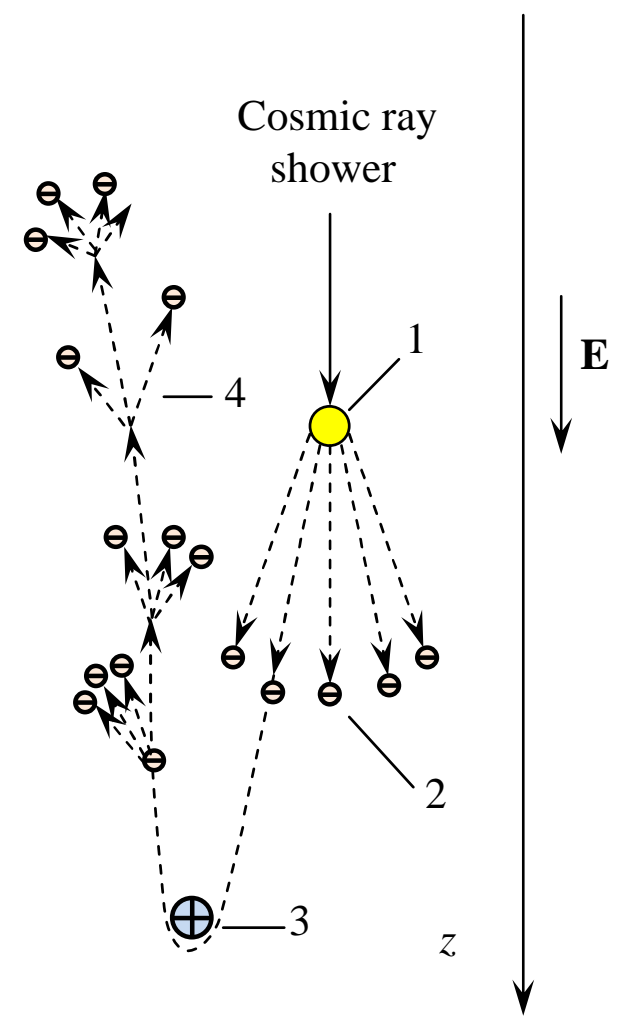

Fig. 4. A schematic illustration of upward avalanche of fast seed electrons resulting from a cosmic ray shower in the presence of the downward-directed ambient electric field $\boldsymbol{E}$. Trajectory of the secondary downward-directed electrons can be distorted due to the scattering by nuclei. 1 - incident primary particle, 2 - secondary fast seed electrons, 3 - nucleus, 4 - upward avalanche of electrons.

quasi-static fields of thundercloud can result in narrow bipolar radio pulses and gamma emission. An indirect observational hint that the EAS can trigger these pulses was provided by Gurevich et al. (1999b).

An alternative mechanism for runaway is based on the electron acceleration during propagation of the lightning streamers and stepped leaders (e.g. Carlson et al., 2010; Chanrion and Neubert, 2010). The numerical simulation has shown that, at the stage of negative corona flash, the exponential growth of potential differences in streamers can give rise to the production of runaway electrons with energies as high as $\sim 100 \mathrm{keV}$ (Celestin and Pasko, 2011). Moreover, the electric field produced by stepped leaders can accelerate those energetic electrons up to the $\mathrm{MeV}$ energies.

The secondary fast electrons may come out from the radioactive decays in air as well. Paiva et al. (2009) have recently assumed that the decay of a rest muon after an intracloud lightning discharge can provide the relativistic electrons. This model predicts $10^{7}$ relativistic seed electrons per $\mathrm{ms}$ at about $15 \mathrm{~km}$ altitude with mean energy of $35 \mathrm{MeV}$.

For the sprite initiation to succeed, the strong QE field must occur over a lightning storm. Essentially for all events, 
the sprite generation is associated with a positive flash (Boccippio et al., 1995; Williams et al., 2007). Just after a positive CG return stroke, the uncompensated negative charge of the thundercloud results in the generation of downwarddirected electric field over the thundercloud. Originally, this electric field decreases the energy of downward-moving fast seed electrons and changes their momentum. The turn of the seed electron trajectories towards the ionosphere can be due to the interaction of the electrons with massive nuclei of molecules as schematically shown in Fig. 4. When the scattering of the seed electrons by nuclei occurs, it produces the electrons moving in all directions. As the field exceeds the breakdown threshold, it can generate the upward-directed runaway electron avalanche, thereby exciting the breakdown of air at stratospheric and mesospheric altitudes.

The mean flux density of the secondary electrons at the thundercloud altitude, that is $4-8 \mathrm{~km}$, is estimated as $10^{3}$ electron $\mathrm{m}^{-2} \mathrm{~s}^{-1}$ (Bazilevskaya and Svirzhevskaya, 1988). The detailed Monte Carlo simulations of the cosmic ray secondary flux distributions of the relevant to relativistic runaway electron avalanche seeding have shown the close value of the flux density about $2 \times 10^{3} \mathrm{~m}^{-2} \mathrm{~s}^{-1}$ at the $15-$ $25 \mathrm{~km}$ altitude range (Carlson et al., 2008). Notice that only a portion of these downward electrons can turn the trajectory to create the upward electron avalanche.

It should be noted that the Earth's magnetic field may greatly affect the motion of relativistic electrons as their gyrofrequency prevails the electron-neutral and ion-neutral collision frequencies. In the atmosphere, $1 \mathrm{MeV}$ electrons become magnetized ones above approximately $20 \mathrm{~km}$ altitude. In such a case, the Earth's magnetic field turns the electron trajectories along the magnetic field lines and weakens the runaway breakdown (Gurevich et al., 1996; Lehtinen et al., 1999; Milikh and Roussel-Dupré, 2010).

An observational hint about the existence of runaway electron breakdown and associated X-ray emissions under high temperature and ionization was provided by Kurzan et al. (1995) and Kuznetsov et al. (1999) using measurements in tokamak. Laboratory tests have shown that long sparks in air are accompanied by a rapid increase in the number of fast electrons with wide energy spectrum $(1-300 \mathrm{keV})$ followed by X-ray bursts and strong flashes of $\gamma$ photons up to a few $\mathrm{MeV}$ possibly related to the runaway breakdown (Gurevich et al., 1999a; Babich et al., 2004; Dwyer et al., 2005; Rahman et al., 2008; Milikh and Roussel-Dupré, 2010). However, these experiments cannot provide us with unambiguous evidence of the leading mechanism of the effect since the applied field was about $1.1 \mathrm{MV} \mathrm{m}^{-1}$, which is smaller than that of conventional breakdown and greater than that of runaway breakdown. At the moment, the laboratory imaging of spatial structure of runaway avalanches has not yet been observed.

The runaway electron mechanism can serve as a promising candidate for explanation of the so-called terrestrial gamma ray flashes (TGFs), that is, short bursts of gamma rays originating from Earth. The TGFs have now been extensively studied for more than a decade, beginning with their modern discovery (Fishman et al., 1994). It is generally believed that the TGFs are associated with an individual lightning strike since they occur in the form of narrow beams within a few ms of the lightning (Fishman et al., 1994; Inan et al., 1996a; Inan and Lehtinen, 2005; Cummer et al., 2005; Smith et al., 2005, 2010; Briggs et al., 2010). The observed rate of TGF events is much smaller than that of lightning flashes maybe due to the fact that it is difficult to detect the TGFs.

Some problems arise because the runaway theory predicts that the lightning charge moment change needs to be about $5000 \mathrm{C} \mathrm{km}$ to generate the runaway breakdown at the altitude range $30-50 \mathrm{~km}$ followed by the TGFs events (Lehtinen et al., 2001). This contradicts with observations since the measured lightning charge moment changes in TGF-associated strokes are 50-500 times smaller than what the runaway theory predicts (Cummer et al., 2005). However, it is still possible that some TGFs are generated by enormous lightning discharges with charge moment changes of several thousands of C km (Stanley et al., 2000; Cummer and Füllekrug, 2001).

The model of TGF production by runaway breakdown in a strong electric field at the tip of lightning leader channels has been recently studied (Trakhtengertz et al., 2002, 2003; Gurevich et al., 2004, 2007; Dwyer, 2008; Dwyer et al., 2010; Carlson et al., 2010; Celestin and Pasko, 2011). Another promising viewpoint on TGFs supposes that the gamma bursts are generated in thunderclouds as a result of the combined action of runaway breakdown and EASs (Gurevich and Zybin, 2004). On the other hand, one should take into account a strong damping of gamma radiation in the lower atmosphere. In this notation, it would be expected that the TGF sources occur most likely at $\sim 10-15 \mathrm{~km}$ altitude. Additionally, one may hypothesize a possibility for transport of the runaway electrons from the source to the desired height of $30-35 \mathrm{~km}$ where the gamma emission due to Bremsstrahlung can escape into space to be detected by on-board sensors.

\section{Underlying mechanisms of transient luminous events}

The foregoing analysis has demonstrated that two different models based on thermal and relativistic electrons, respectively, can explain, in principle, the molecular ionization in an electrically heated ionized gas. As would be expected, the same approaches can be applied to the process of air breakdown above a lightning storm at stratospheric and mesospheric altitudes.

\subsection{Effect of charge distribution in thunderclouds on jet discharges}

Most of the BJ events are analogous in character to propagation of positive leader of conventional breakdown, whereas the majority of GJs manifest themselves as a negative leaderlike propagation (Krehbiel et al., 2008). Other types of high 


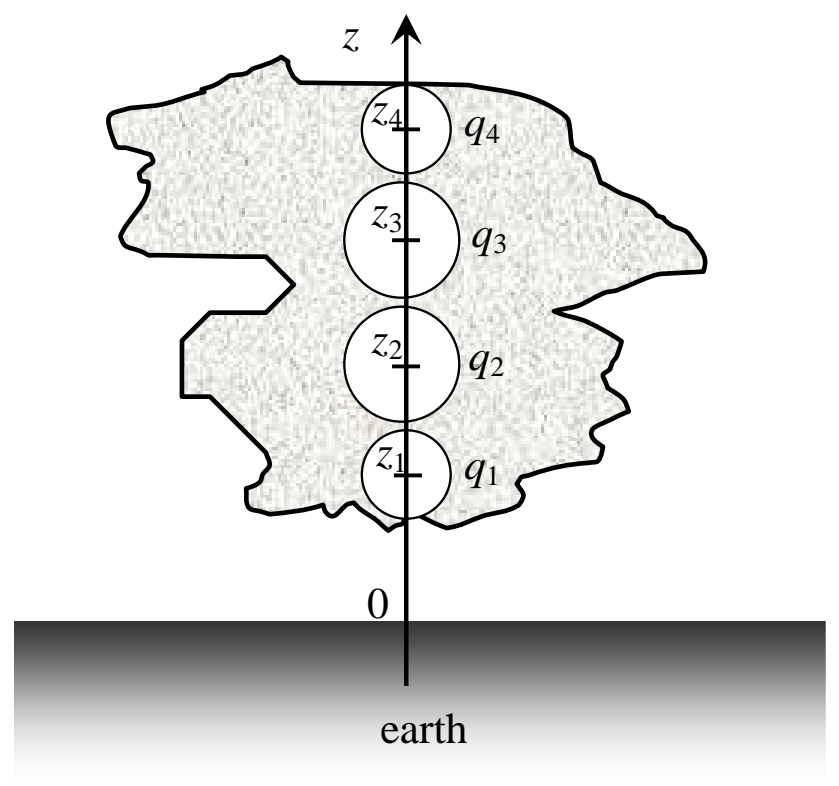

Fig. 5. Simplified model of electric charge distribution in a thundercloud. The spatial charge densities are constant inside the spherical regions whose centers are located on the $\mathrm{z}$-axis at different altitudes $z_{1}, z_{2}, z_{3}$ and $z_{4}$.

altitude discharges, i.e. sprites, usually take place just after intense positive cloud-to-ground strokes followed by the formation of a large amount of uncompensated negative cloud charges (Boccippio et al., 1995; Williams, 2007). In contrast to sprites, the thunderstorm charge redistribution due to the stroke appears to have no effect on the generation of blue and GJs.

The BJs are supposed to occur under certain relatively rare conditions, when large positive charge piles up at the top of thundercloud. The negative charges at the bottom of thundercloud compensate, in part, the field of positive charges in the region just above the thundercloud. However, local inhomogeneities of the spatial charge distribution at the top of thundercloud may result in the enhancement of electric field in this region.

The presence of the strong QE field above the thundercloud is extremely important in the modeling of streamer type processes at stratospheric and mesospheric amplitudes. For example, in the early model of the blue jet (Pasko et al., 1996a), the positive charges were assumed to reach a total value about $300-400 \mathrm{C}$ as high as $15-20 \mathrm{~km}$ altitude. As another example, in the 3-D model by Pasko and George (2002), it was assumed that a positive charge of 100-150 C can accumulate at altitude about $15 \mathrm{~km}$ to provide the electric field magnitude capable of exceeding $E_{\mathrm{cr}}^{+}$threshold just above the thundercloud.

Notice that the electrical structure/charge distribution of actual thundercloud is much more complicated as compared to the modeling. Moreover, a certain charge imbal- ance may persist in thunderstorm, which causes strong variations of the electric field with altitude. Recently, Krehbiel et al. (2008) and Riousset et al. (2010b) have discussed a stratiform/multilayered thundercloud model to demonstrate the difference between the conditions for downward-propagating discharges in the form of usual -CG lightning and for upward-propagating discharges like jets. The charged regions situated at different altitudes in the thundercloud are sketched in Fig. 5 with white circles. For example, a normally electrified storm, which corresponds to typical -CG lightning, was characterized by the following numerical values: $q_{\mathrm{i}}=12.5,-60,40,-20 \mathrm{C}$, where $i=1,2,3,4$ (Krehbiel et al., 2008). The spatial distribution of these charges was assumed to obey Gaussian low and was not spherically symmetric.

To simplify the problem and to interpret the results by Krehbiel et al. (2008), we assume that all the charges are uniformly distributed in spherical regions shown in Fig. 5. The earth is considered to be a perfect conductor. This implies that the vertical component of the net electric field taken along the z-axis is given by $E_{\mathrm{z}}=\sum_{i=1}^{4} E_{\mathrm{zi}}$, where

$E_{\mathrm{zi}}=k q_{\mathrm{i}}\left(z-z_{\mathrm{i}}\right) / r_{\mathrm{i}}^{3}+E_{\mathrm{zi}}^{\prime}$

inside the charged balls and

$E_{\mathrm{zi}}=k q_{\mathrm{i}}\left(z-z_{\mathrm{i}}\right) /\left|z-z_{\mathrm{i}}\right|^{3}+E_{\mathrm{zi}}^{\prime}$

outside the balls. Here $E_{\mathrm{zi}}^{\prime}=k q_{\mathrm{i}} /\left(z+z_{\mathrm{i}}\right)^{2}$ stands for the electric field of mirror electric images of thundercloud charges, $k=\left(4 \pi \varepsilon_{0}\right)^{-1}, \varepsilon_{0}$ is the electric constant/permittivity of vacuum, and $z_{\mathrm{i}}$ and $r_{\mathrm{i}}$ denote coordinates of centers and radii of the charged spherical regions, respectively. The variations of the net electric field, $E_{\mathrm{z}}$, as a function of altitude $z$ can be easily calculated after these simplifications.

To illustrate the results of this simulation, we made use of the following numerical parameters: $z_{\mathrm{i}}=3.7,6.9,12.1,15.7 \mathrm{~km}$, and $r_{\mathrm{i}}=1.0,2.2,2.3,1.3 \mathrm{~km}$ and above-mentioned values of $q_{\mathrm{i}}$ taken from Krehbiel et al. (2008). The results of calculations shown in Fig. 6 with solid line are in qualitative agreement with the vertical profiles of thunderstorm electric field as measured by balloon equipment (Marshall et al., 1995). The first two peaks at the bottom of Fig. 6 are basically due to the field of pair of charges $q_{1}=12.5 \mathrm{C}$ and $q_{2}=-60 \mathrm{C}$, whereas the two peaks at the top of Fig. 6 are caused by the upper charges $q_{3}=40 \mathrm{C}$ and $q_{4}=-20 \mathrm{C}$. It should be noted that both the magnitude and location of the peaks are very sensitive to the distances between the charges.

The lightning leader propagation is accompanied by the streamer zone formation. The electric field required for the propagation of negative and positive streamers in the air is shown in Fig. 6 with dashed lines 2 and 3. The runaway 


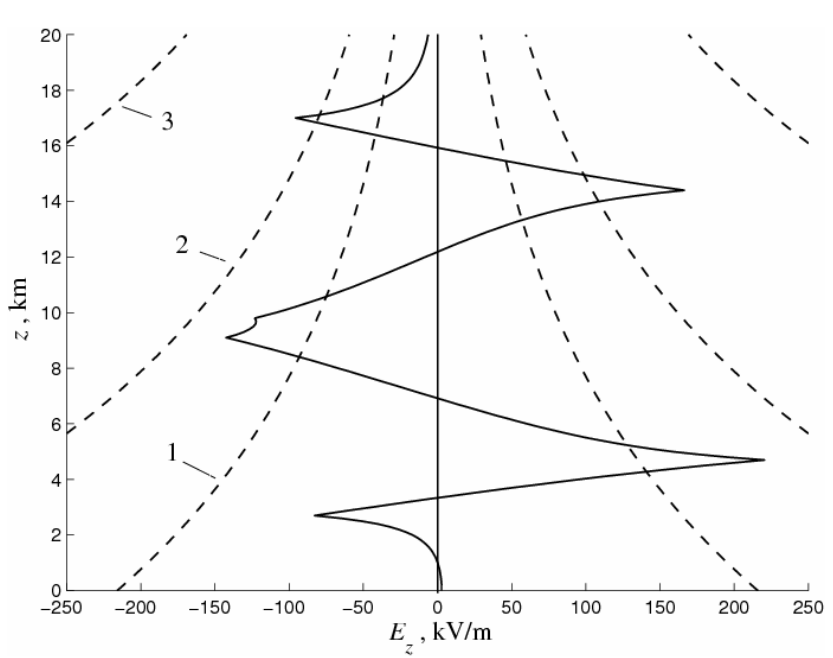

Fig. 6. Model calculations of thunderstorm QE field preceding conventional -CG stroke. The vertical electrical field profile along zaxis as a function of altitude $z$ is shown with solid line. A runaway breakdown field is shown with dashed line 1, while the electric fields required for propagation of negative and positive streamers in the air are shown with dashed lines 2 and 3 . The numerical values of parameters are assumed to be typical for generation of $-\mathrm{CG}$ strokes.

breakdown field (Eq. 24) is shown with dashed line 1. The same lines are shown symmetrically on the right of Fig. 6. Despite that the calculated value of electric field does not exceed the breakdown threshold for streamer propagation, the $-\mathrm{CG}$ may be initiated in the vicinity of the first peak at 4-6 km altitude range due to presence of spatial inhomogeneities of the charge distribution that may result in the local enhancement of the field. Notice that the runaway breakdown can serve as another trigger mechanism for the streamer initiation since the runaway threshold (line 1) is the nearest to the peak values of thunderstorm electric field (e.g. see Milikh and Roussel-Dupré, 2010).

A BJ may occur under certain meteorological conditions when large amount of positive charge is concentrated at the top of thundercloud. To simulate a normal blue jet discharge in the framework of the stratified thunderstorm model, Krehbiel et al. (2008) have suggested the set of parameters $q_{\mathrm{i}}=5,-40,57.5,-20 \mathrm{C}$. In Fig. 7, we plot the numerical calculation of the vertical field versus altitude based on the simplified model shown in Fig. 5. In making the plot of $E_{\mathrm{z}}$, we have used the same parameters $z_{\mathrm{i}}$ and $r_{\mathrm{i}}$ as in Fig. 6. It is obvious from this figure that the positive peak of $E_{\mathrm{Z}}$ exceeds $E_{\mathrm{cr}}^{-}$(line 2) and becomes close to $E_{\mathrm{cr}}^{+}$(line 3) at $z \approx 14 \mathrm{~km}$. On account of the field direction at this altitude, it would be expected that the upward-directed positive leader originating at this point can propagate towards the ionosphere.

It is generally accepted that the GJ events should be associated with great deal of negative electric charges situated at the middle region of thundercloud. Typical parameters,

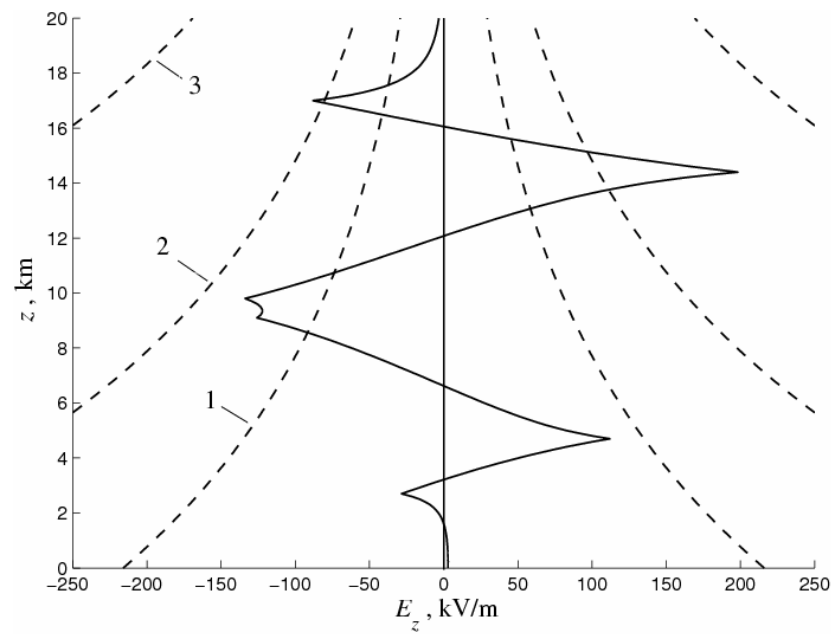

Fig. 7. Model calculations of thunderstorm QE field preceding blue jet discharge.

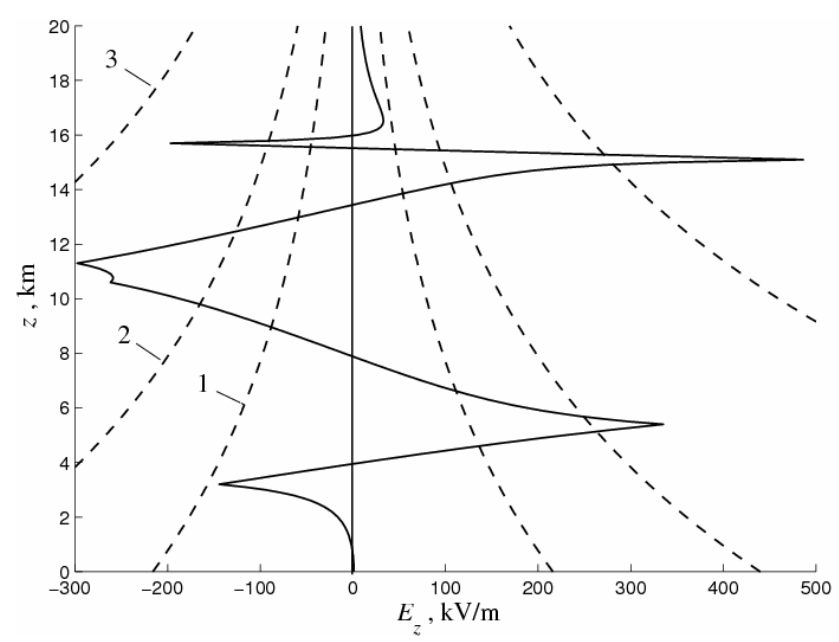

Fig. 8. Model calculations of thunderstorm QE field preceding gigantic jet discharge.

which correspond to this situation, are as follows (Krehbiel et al., 2008): $q_{\mathrm{i}}=25,-120,82.5,-3 \mathrm{C}$. Figure 8 shows the model calculations of $\mathrm{QE}$ field preceding generation of gigantic jet. The numerical values of parameters used in making this plot are as follows: $z_{\mathrm{i}}=4.3,8.0,13.2,15.4 \mathrm{~km}$ and $r_{\mathrm{i}}=1.1,2.6,1.9,0.3 \mathrm{~km}$. As is seen from this figure, the GJ can develop originally as an upward-propagating intracloud discharge originating from the site situated above the charge $q_{2}=-120 \mathrm{C}$ within the altitude range $10-12 \mathrm{~km}$ where the thunderstorm electric field exceeds the breakdown threshold. The jet current bears a negative charge of the order of $100 \mathrm{C}$ through the region with upward/positive electric field (above the charge $q_{3}=82.5 \mathrm{C}$, the altitudes $13-15 \mathrm{~km}$ ) towards the thundercloud top. One may assume that the GJ can overcome the region with the positive field to propagate out of the thundercloud towards the ionosphere (Krehbiel et al., 
2008; Pasko, 2010). However, we cannot explain in any detail why the gigantic jets look more powerful than the blue jets and why they can extend to higher altitudes.

\subsection{Blue jets and gigantic jets}

Typically, the BJ has a conical shape whose transverse size increases from $1-2 \mathrm{~km}$ up to several kilometers in the $15-50 \mathrm{~km}$ altitude range. In early theories of BJ, Pasko et al. (1996a) and Sukhorukov et al. (1996a, b) considered the $\mathrm{BJ}$ as a mesospheric analog of upward-propagating positive/negative streamer similar to that observed at the laboratory conditions. A possible scenario of BJ evolution shown in Fig. 1 assumes that the BJ originates from a sharp positively charged irregularity situated at the top of the thundercloud (Pasko et al., 1996a; Pasko and George, 2002). Such irregularity with lateral extent about $10^{2}-10^{3} \mathrm{~m}$ can serve as an analog of the point electrode in laboratory point-to-plane corona discharge experiments. The head of jet streamer bears a positive charge to the conducting electrosphere, which plays a role of the plane electrode.

These models assume initial transverse size of the streamer of about $1 \mathrm{~km}$ that contradicts with estimates of the streamer size based on similarity law (Pasko, 2007, 2010). According to this law, the transverse size of typical streamer should be of the order of several centimeters at $30 \mathrm{~km}$ altitude where blue jets usually occur. The BJ modeling is further complicated by the fact that below $40 \mathrm{~km}$ the streamer conductivity falls off faster due to electron attachment to the atmosphere oxygen in triple collisions according to reaction (5). Additionally, the jet streamer lifetime $(\sim 1 \mathrm{~s})$ contradicts with the short decay time of cold plasma in streamer channel $(\sim 10 \mu \mathrm{s})$ because the jet streamers cannot be supplied with current during their lifetime (Raizer et al., 2010).

In later work, Petrov and Petrova (1999) proposed a more realistic model in which $\mathrm{BJ}$ is treated as upward-propagating positive leader with a streamer zone/corona on the top as schematically displayed in Fig. 1. The leader transfers a positive charge from the thundercloud up to altitude about several tens of kilometers at the velocity much slower than that of individual streamers. A great number of the short-lived streamers are emitted from the leader thereby producing a streamer corona. A majority of the streamers are assumed to be upward directed to produce a branching structure of BJs, which is consistent with the observations. A conical shape of BJs follows the similarity law (Eq. 17) according to which the scale of individual streamers and of the whole streamer zone has to increase with height.

The streamer-to-leader transition is accompanied by the Joule heating and subsequent detachment processes (Bondiou and Gallimberti, 1994). In this picture the BJs are similar to hot leader-like discharges rather than cold streamer-like discharges (Raizer et al., 2010).

Considering the streamer zone, we note that our calculations have demonstrated that the electric field required for propagation of positive streamer is still greater than the field caused by thundercloud charges (Fig. 7). This leads us to the conclusion that any kind of electrical inhomogeneity needs to start breakdown ionization of the air. Following Pasko et al. (1996a) and Pasko and George (2002), one may suppose that the electric irregularity/overcharge about $10 \mathrm{C}$ with characteristic radius about $500 \mathrm{~m}$ occurs in the upper portion of thundercloud; then, the electric field reaches a value about $400 \mathrm{kV} \mathrm{m}^{-1}$ in the vicinity of this irregularity. As is seen from Fig. 7, this value is greater than threshold for positive streamer (line 3 ) and it is the same order as the conventional breakdown threshold taken at the altitude $15-20 \mathrm{~km}$. However, such a large value of the thundercloud charges that is required to sustain the $\mathrm{BJ}$ seems to be well-nigh impossible (Mishin and Milikh, 2008; Raizer et al., 2010).

Raizer et al. $(2006,2007)$ have supposed that BJ can be the result of the formation of a bidirectional uncharged leader at the point where the electric field reaches a maximum value inside the thundercloud (see positive peak shown in Fig. 7). Owing to the exponential profile of the air density, the streamers are assumed to grow predominantly upward in contrast to laboratory conditions.

The gigantic jets are believed to be due to the predominance of negative charges at the top of thundercloud as shown in Fig. 7. Since the gigantic jets show up the visible patterns similar to inverted images of conventional - $\mathrm{CG}$, it appears that the most of gigantic jets develop in the form of upwardpropagating negative leaders (Pasko, 2010). For example, a gigantic jet recently observed by Cummer et al. (2009) was estimated to transfer the negative charge of $-144 \mathrm{C}$ from the thundercloud to the lower ionosphere. However, the onset time of the current due to gigantic jet is about $30 \mathrm{~ms}$, that is, much greater than that ( $\sim 5 \mu \mathrm{s})$ (Rakov and Uman, 2003) due to normal -CG.

In contrast to the early study, the first documented GJ, which can be referred to as the class of positive cloud-toionosphere discharge $(+\mathrm{CI})$, has been recently observed by van der Velde et al. (2010) during winter thunderstorms in the Mediterranean. Despite the short duration (120-160 ms), this GJ is estimated to transfer negative charge $\sim 136 \mathrm{C}$ from the ionosphere to the positively charged origins in the cloud only $6.5 \mathrm{~km}$ tall, showing high altitudes are not required for initiation of GJs. This event is characterized by a huge charge moment change of $11600 \mathrm{C} \mathrm{km}$ and a maximum GJ current of $3.3 \mathrm{kA}$ (van der Velde et al., 2010).

In conclusion we note that, although the similarity law for leader does not exist (Raizer, 1991), with some care the above qualitative estimates given in Sect. 2.3 are applicable not only to the streamer scales but also to the scales of jet streamer zone as whole. In particular, one may expect that the streamer zone radius as well as the cross section of BJs and GJs will increase with altitude in accordance with estimate given by Eq. (17). This simplified model can explain, in principle, a blue conical shape of jets, which was well documented by airplane and ground-based observations (Wescott 
et al., 1995; Neubert et al., 2008). Certainly the numerical estimates of the BJs and GJs are quite different, because the minimum value of electric field required for the propagation of negative streamers in the air is greater than the field, which is necessary to trigger positive streamers (Raizer, 1991). It appears that the typical size, velocity and other parameters of BJs and GJs can be very sensitive to the initial conditions at the top of thundercloud such as the charge distribution shown in Fig. 5 and rate of charge accumulation.

An analytical solution describing the jet evolution has not been found yet except for the simple similarity relationships (Raizer et al., 2010).

\subsection{Sprites}

Worldwide sprites and halos are predominantly excited due to large CG flashes almost exclusively with positive polarity (e.g. Boccippio et al., 1995; Williams et al., 2007). The sprites and mesospheric TLEs occur infrequently since the charge moment change of the causative +CG must be more than a value of the order of $500 \mathrm{C} \mathrm{km}$ in order to initiate the sprite discharge (Stanley et al., 2000; Cummer, 2003; Rycroft, 2006; Hiraki and Fukunishi, 2006). The point of sprite initiation occurs in the $60-80 \mathrm{~km}$ altitude range, while the brightest region of the sprite body is usually observed in the altitude range $65-85 \mathrm{~km}$ (Neubert et al., 2008).

The high-speed video recording of sprites has shown the streamer-like pattern of the sprite discharges (Cummer et al., 2006b; Stenbaek-Nielsen and McHarg, 2008; Liu et al., 2009; Montanyá et al., 2010). It is generally accepted that there are two basic visible shapes of sprites: "carrot" or "jellyfish" configuration and columniform (e.g. Cho and Rycroft, 1998; Matsudo et al., 2007). The carrot type sprite contains diffuse tops and lower tendrils extending down to altitudes of 30-40 km, while the columniform sprite has a very fine spatial structure as compared with the "carrot" sprite (e.g. Hayakawa et al., 2004; Wescott et al., 1998). The faint red glow diffusion region lies in the altitude range 85 to $90 \mathrm{~km}$, while the brightest region occurs in the $65-85 \mathrm{~km}$ altitude range. The sprite measurements during winter thunderstorms in the Hokuriku area of Japan have shown that the "column" sprites occur several ms after an intense +CG discharge ( 100 kA), while "carrot" sprites might be delayed by some tens of ms after a $+\mathrm{CG}$ discharge (e.g. Matsudo et al., 2009). It appears that the "carrot" sprites are associated with a smaller $+\mathrm{CG}$ discharge $(\sim 30 \mathrm{kA})$ but with a strong continuing current (Neubert et al., 2008). A few active thunderstorms and thunderstorms with precipitating stratiform clouds may excite more complex sprites possibly associated with the CC of the causative lightning (Suzuki et al., 2011).

Recent studies of the sprite structure based on the high speed camera observations have shown that columnar sprites arise from downward propagating streamers, while the carrot sprites start as columnar sprites followed by upward propagating streamers (McHarg et al., 2007; Stenbaek-Nielsen and McHarg, 2008). The branches are the trail afterglow of the upward and downward propagating streamer heads which manifest themselves as very bright small formations. As for the sprite morphology, this study has demonstrated that columnar and carrot sprites cannot be split into two individual classes.

The positive CG flashes preceding the sprite initiation may result in the extraordinary large charge transfer of the order of hundreds of coulombs (Uman, 1987; Rakov, 2000; Rakov and Uman, 2003). The positive charges can disappear for the short time about $1 \mu$ s that gives rise to the strong quasistationary electric field caused by negative charges predominantly located at the bottom of the thundercloud. The electric field may exceed the breakdown threshold between the thundercloud top and the ionosphere. Below 50-60 km altitude, the field relaxation time $\tau=\varepsilon_{0} / \sigma_{\mathrm{a}}$ due to air conductivity, $\sigma_{\mathrm{a}}$, is about or greater than $0.1-1 \mathrm{~s}$, which makes it possible to generate a sprite for that period.

Pasko et al. (1998a) and Pasko (2010) have assumed that the "carrot" sprite morphology and spatial structure can be explained in terms of the conventional breakdown theory and three physical timescales. The first timescale, $t_{\mathrm{s}}$, is the time necessary for development of the streamer zone that may cause the relaxation of the applied electric field. In the highly structured lower region below $75 \mathrm{~km}$, it is conjectured that $t_{\mathrm{s}}<\tau=\varepsilon_{0} / \sigma_{\mathrm{a}}$; that is, the streamer mechanism of the field amplification prevails over the field relaxation due to conductivity. The diffusion zone $\left(t_{\mathrm{s}}>\tau\right)$ above $85 \mathrm{~km}$ is assumed to be due to the usual Townsend electron multiplication mechanism. The transition zone $\left(t_{\mathrm{S}} \sim \tau\right)$ sandwiched between the diffuse and streamer regions in the 75-85 altitude range as well as the streamer zone is assumed to be characterized by strong attachment of ambient electrons so that the dissociative attachment timescale $\left(v_{a}-v_{i}\right)^{-1}$ is smaller than $\tau$. The boundaries of the transition zone can shift depending on the condition for the streamer generation (Stanley et al., 1999; Pasko, 2006; McHarg et al., 2007).

Consider first a short period $\Delta t \ll \tau$ just after the causative $+\mathrm{CG}$ lightning. This implies that the conduction current, $\sigma_{\mathrm{a}} E$, is much smaller than the displacement current, $\varepsilon_{0} \partial E / \partial t$, so that the air conductivity can be neglected in the first approximation. Since we study the electric field at sprite altitude beyond thundercloud, the actual charge distribution in the thundercloud is of no importance. The uncompensated/unbalanced negative charges arising in the thundercloud just after $+\mathrm{CG}$ are assumed to be uniformly distributed inside the ball with radius $r$. The center of the charged ball is located on z-axis at the altitude $h$. The electric field of these charges and of their electrical images in the perfectly conducting ground is described by equations similar to Eqs. (29) and (30). The calculated electric fields $E_{\mathrm{z}}$ on z-axis at the numerical values $h=10 \mathrm{~km}$ and $r=2 \mathrm{~km}$ are shown in Fig. 9 with lines $1-3$, which correspond to $q=50,100$ and $150 \mathrm{C}$, respectively. Notice that the initial charge $q$ can gradually increase just after the main stroke due to strong continuing 


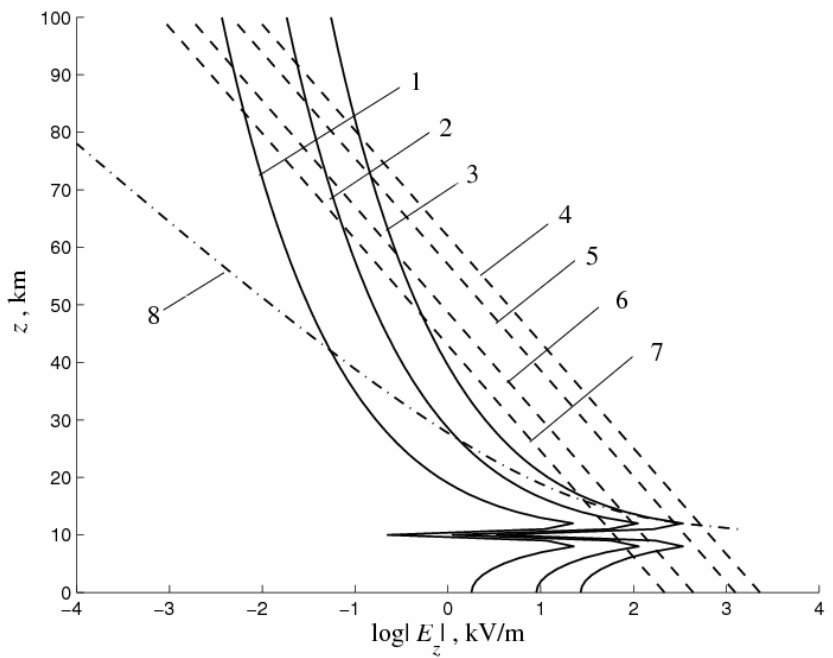

Fig. 9. Model calculations of thunderstorm QE field preceding sprite discharge. Absolute value of the vertical electric field is shown with lines $1-3$, which correspond to the thunderstorm charge $q=50100$ and $150 \mathrm{C}$, respectively. In making these plots, the air conductivity was ignored. Altitude dependences of breakdown electric field that correspond to different air breakdown mechanisms are shown with dotted lines: 4 - conventional breakdown threshold, 5 - negative streamer propagation, 6 - positive streamer propagation, 7 - relativistic runaway breakdown. Dashed-and-dotted line 8 illustrates the effect of atmospheric conductivity, which obeys exponential law (Eq. 31), on thunderstorm QE field. This plot corresponds to the thunderstorm charge $q=150 \mathrm{C}$.

current (CC) in sprite-associated $+\mathrm{CG}$ lightning. This $\mathrm{CC}$ is normally much greater than that due to negative stroke, and its value amounts to $5-10 \mathrm{kA}$ for a period $10-100 \mathrm{~ms}$ (Rakov, 2000). In this notation, the lines $1-3$ can illustrate the enhancement of QE thunderstorm field that resulted from the thunderstorm charge accumulation during the $\mathrm{CC}$.

We recall that the electron avalanches caused by thermal ionization can develop under condition that the electric field exceeds the conventional breakdown threshold $E_{\mathrm{k}}$, whereas the streamer may propagate in the electric field lower than $E_{\mathrm{k}}$. All the threshold fields exponentially decrease with altitude. The conventional breakdown threshold is plotted in Fig. 9 with dotted line 4, while the propagation thresholds for positively and negatively charged streamers are shown with dotted lines 5 and 6 . The threshold for runway breakdown is shown in Fig. 9 with dotted line 7.

It is obvious from Fig. 9 that the electric field of the thunderstorm can exceed all the breakdown thresholds in the altitude range $50-80 \mathrm{~km}$ (for example, see line 3). This result is readily apparent from the fact that the dipole electric field caused by the thundercloud charge and its electric image in the conducting ground falls off inversely as the cube of the distance from the source, whereas the breakdown field falls off exponentially with altitude, that is, more rapidly than does the thunderstorm electric field. So, there may be a height above which the thundercloud electric field exceeds the breakdown threshold (Wilson, 1925). As is seen from Fig. 9, this situation may exist at the mesospheric altitude range.

An exponential increase of the atmospheric conductivity with altitude may greatly affect the sprite formation, which is likely to proceed at mesospheric altitudes. The generation of QE electric fields above a thundercloud is restricted by the relaxation process due to air conductivity. The latter depends on the ionization caused by cosmic rays, the ion-neutral collision rate etc., which in turn exponentially decreases with altitude due to changes of the air density. Notice that the air conductivity is mainly due to the presence of ions since free electrons are captured by molecules for a short time about $0.1 \mu$ s at the ground level (e.g. see reactions given by Eqs. 3 and 5). The air conductivity as a function of altitude $z$ can be approximated via

$\sigma_{\mathrm{a}}=\sigma_{0} \exp (\alpha z)$

where $\alpha$ is a constant and $\sigma_{0}$ denotes the conductivity of the standard atmosphere at the ground level.

Considering the thundercloud as a point current source located on z-axis at the altitude $h$, we assume that the time scale of the source variations is much greater than the relaxation time, that is, $\Delta t \gg \tau$. In this extreme case, a distribution of electric potential $\varphi$ is described by Poisson equation. Taking into account an axial symmetry of the problem, using cylindrical coordinates $z, r$, and with assumption that variations of $\sigma_{\mathrm{a}}$ occur only in the z-direction, the Poisson equation reads

$\frac{\sigma_{\mathrm{a}}}{r} \frac{\partial}{\partial r}\left(r \frac{\partial \varphi}{\partial r}\right)+\frac{\partial}{\partial z}\left(\sigma_{\mathrm{a}} \frac{\partial \varphi}{\partial z}\right)=\frac{I \delta\left(r_{0}\right)}{4 \pi r_{0}^{2}}$,

where $r_{0}=\left\{r^{2}+(z-h)^{2}\right\}^{1 / 2}$. Here $\delta$ stands for Dirac deltafunction and $I$ denotes the total current flowing from the source.

This equation should be supplemented by the proper boundary conditions for the conducting ground and at the infinity, that is, $\varphi=0$ at $z=0$ and $\varphi \rightarrow 0$ when $z \rightarrow \infty$. The solution of this problem is given by (e.g. see Soloviev and Surkov, 2000)

$$
\begin{aligned}
\phi= & \frac{I}{4 \pi \sigma_{0}} \exp \left[-\frac{\alpha}{2}(z+h)\right] \\
& \left\{\frac{1}{r_{0}} \exp \left(-\frac{\alpha r_{0}}{2}\right)-\frac{1}{r_{1}} \exp \left(-\frac{\alpha r_{1}}{2}\right)\right\},
\end{aligned}
$$

where $r_{1}=\left\{r^{2}+(z+h)^{2}\right\}^{1 / 2}$. The electric field is derivable from a potential $\varphi$ through $\boldsymbol{E}=-\nabla \varphi$. The total charge $q$ of the source/thundercloud can be related to the source current $I$ through Gauss theorem and Ohm law; whence it appears that $I=\left(q \sigma_{0} / \varepsilon_{0}\right) \exp (\alpha h)$.

The exponential factors in Eq. (33) describe the field attenuation due to air conductivity. Indeed, when $\alpha \rightarrow 0$, the potential (Eq. 33) corresponds to a field of point charge $q$ and its 
mirror image in the conducting ground. A model calculation of the vertical component of electric field along $\mathrm{z}$-axis in the presence of atmospheric conductivity is shown in Fig. 9 with dashed-and-dotted line 8. In making the plot of $E_{\mathrm{z}}$, we have used the numerical values $q=150 \mathrm{C}$ and $\alpha \approx 0.15 \mathrm{~km}^{-1}$.

It is obvious from Fig. 9 that the conduction current due to the atmospheric conductivity may decrease the thunderstorm field to such an extent that it makes impossible the air breakdown in the mesosphere. Certainly the above considered extreme case cannot provide us with detailed information about effect of the air conductivity on the evolution of the thunderstorm QE field. In a more accurate model, the electric field shape in the continuing current stage can be situated between lines 3 and 8 (Mareev and Trakhtengerts, 2007).

The electron associative detachment from atomic oxygen ions (Eq. 6) is capable of explaining the electron production under electric field below the conventional breakdown field. Since the increase of thundercloud electric field is accompanied by impact ionization and electron dissociative attachment according to Eqs. (2) and (3), the $\mathrm{O}^{-}$ions may pile up at the upper ionosphere. It should be noted that $\mathrm{O}^{-}$ions are short-lived, because they are attached to oxygen $\mathrm{O}_{2}$ by a three-body clusterization reaction thereby producing much more stable ozone ions $\mathrm{O}_{3}^{-}$. Assuming that this reaction is negligible during tens of milliseconds, Luque and GordilloVázquez (2012) have shown that the number density of $\mathrm{O}^{-}$ ions can be high enough to produce free electrons due to associative detachment (Eq. 6), which in turn may result in breakdown of air.

Figure 10a displays a model of the carrot-like or jelly-fishlike sprite evolution after +CG lightning (Raizer et al., 2010). The sprites build up very quickly just after the causative lightning discharge for the short period limited by the relaxation time $\tau$ that varies within $1-100 \mathrm{~ms}$ in the altitude range $60-80 \mathrm{~km}$. At first the initial downward-propagating positive streamer is launched from a diffuse glow, termed a halo, as observed by Cummer et al. (2006b). Several milliseconds after this initial stage, a number of new streamers/filaments begin to propagate downward, and branch as they propagate downward, thereby exciting the main flash of the sprite (Stenbaek-Nielsen and McHarg et al., 2008). The tips of the streamers are attracted to and can collide with adjacent streamer channels (Cummer et al., 2006b). At the final stage, the downward-propagating streamers terminate at the altitudes $40-50 \mathrm{~km}$ because of increase of the electric field required for the streamer propagation. The numerical simulations based on this model have been reported by Raizer et al. (2010).

As we have noted above, the sprite morphology includes not only carrot-like sprites but also column and intermediate sprite structure (e.g. see Myokei et al., 2009). In this notation the scenario of sprite development shown in Fig. 10a cannot describe all observations. For example, Cummer et al. (2006a, b) have reported high speed video observations of TLEs originated from brightening inhomogeneities at the
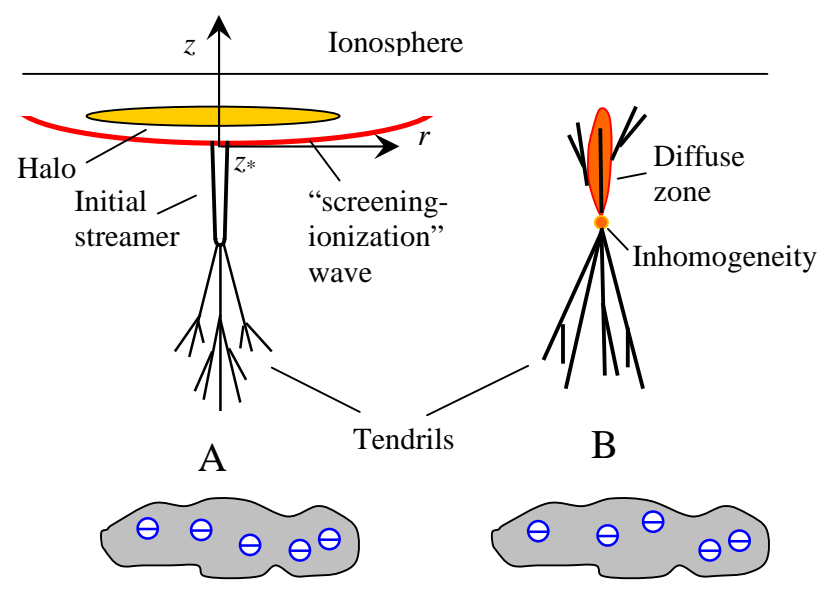

Earth

Fig. 10. A schematic plot of sprite originated from a sprite halo (A) and from an inhomogeneity $(\mathbf{B})$ in the presence of quasi-static electric field generated above thundercloud after $+\mathrm{CG}$ lightning.

bottom of a halo. Figure 10b displays schematic of the sprite evolution according to observations by Cummer et al. (2006b). At first a bright vertical column begins to expand simultaneously upward and downward from the inhomogeneities. This expansion was followed by the development of bright upward-propagating streamers terminated in diffuse emissions and down-propagating streamers that form the bright core of the sprite and tendrils.

It appears that not only the sprite halo but also different kinds of a plasma patch can serve as sprite nucleus depending on meteorological and seasonal conditions, proximity to ocean or land and etc. (e.g. see Myokei et al., 2009; Montanyá et al., 2010). This demands the search for physical mechanism for such patch formation (Raizer et al., 2010).

The sprite origination, visible evolution and their relationship with intra-cloud process treated here are so complex that quantitative theory of the sprites has not been established yet except for a number of numerical simulations. In an early study, the fluid plasma model and conventional breakdown threshold as a critical value for electric field have been used for numerical simulation of the sprite behavior (e.g. Pasko et al., 1998b; Veronis et al., 1999; BarringtonLeigh et al., 2001; van der Velde et al., 2006, 2007; Asano et al., 2009a, b). Pasko et al. $(2000,2001)$ have developed 2-D and 3-D fractal models to describe the lower, highly structured sprite region. In these models, the sprite body builds up as a result of simultaneous upward-propagating negative and downward-propagating positive streamers coming from the same point (see Fig. 10b). In many instances, this scenario is compatible in character with the observation of initial stage of sprite development (Stanley et al., 1999; Stenbaek- 
Nielsen et al., 2000, 2007; Moudry et al., 2003; Cummer et al., 2006b; McHarg et al., 2007; Li and Cummer, 2009).

The detailed calculations of the optical spectrum generated by a sprite are in good agreement with the early video observations and photometric and spectral measurements of sprites (Pasko et al., 1996b; Milikh et al., 1997). Nevertheless, these calculations are based on averaged/simplified distributions of ionized plasma constituents inside the sprite. The recent high-resolution measurements have shown that the largest portion of the optical emission comes from the high-ionized streamer heads which manifest themselves as mobile bright compact balls (Liu and Pasko, 2006; McHarg et al., 2007).

Luque and Ebert $(2009,2010)$ have developed a numerical discharge model, which takes into account Eq. (1) including photoionization effect and altitude-dependent transport and ionization parameters of electrons and neutrals given by Eqs. (10) and (11). The actual charged thunderstorm cloud arising due to $+\mathrm{CG}$ was approximated with a point charge increasing linearly in time. This model does not require any kind of seed electrons since the sprite origin is assumed to be due to drift of the background electrons under the influence of QE field. This implies that the initial sprite firstly arises from the region of sprite halo ( $70-85 \mathrm{~km}$ altitudes) similar to that shown in Fig. 10a. Their numerical simulations show that (1) several ms after the causative +CG a downwardpropagating electron density shock wave can develop in the lower ionosphere; (2) this wave can sharpen and thus transform into the positive sprite streamer as it propagates farther down, and (3) the photo-ionization in the vicinity of streamer's front, where the impact ionization is the highest, could play an important role for the streamer propagation. This electron density shock wave, termed as screeningionization wave, is considered as a possible candidate for the visible sprite halo.

It was suggested by Luque and Ebert $(2009,2010)$ and by Luque and Gordillo-Vázquez (2011) that the enhanced electric field and the vertical gradient of the air density around the streamer head may result in formation of upwardpropagating negative streamer as well as the attraction of downward-propagating sprite streamers to a neighboring channel as observed by Cummer et al. (2006b) and StenbaekNielsen and McHarg (2008) in high-speed observations.

To interpret the results of numerical calculations shown in Figs. 1 and 2 in paper by Luque and Ebert (2009), we assume that the electric field at the front of screening-ionization wave is close to the breakdown threshold given by Eq. (12). Away from the charged thundercloud, the electric field of the thundercloud and its mirror image arising in the perfect conducting ground can be considered in dipole approximation:

$E=\frac{k p}{\left(r^{2}+z^{2}\right)^{3 / 2}}\left(1+\frac{3 z^{2}}{r^{2}+z^{2}}\right)^{1 / 2}$ where $r$ and $z$ are cylindrical coordinates shown in Fig. 10a. The dipole moment $p=2 Q L$, where $Q$ is the thunderstorm charge and $L$ is the distance from the thundercloud to the ground.

We set this field equal to the breakdown threshold (Eq. 12). In this approach, the electric field of charges accumulated at the wave front is ignored. Taking into account Eq. (4), this results in the implicit dependence $r(z)$ or $z(r)$, which defines the surface/line of the wave front. This line crosses z-axes at the point $z_{*}$ that can be found from the following equation:

$\frac{2 k p}{z_{*}^{3}}=A \exp \left(-\frac{z_{*}}{h}\right), A=\frac{\left(E_{\mathrm{i} 0}-E_{\mathrm{a} 0}\right)}{\ln \left(\alpha_{\mathrm{i} 0} / \alpha_{\mathrm{a} 0}\right)}$.

To simplify the problem, we consider the case $r \ll z$, which is valid around the axis of symmetry. In this case, we come to the explicit dependence: $\left(z \geq z_{*}\right)$

$r=2 z\left\{\frac{A}{15 k p}\left[z_{*}^{3} \exp \left(-\frac{z_{*}}{h}\right)-z^{3} \exp \left(-\frac{z}{h}\right)\right]\right\}^{1 / 2}$.

The wave front given by Eq. (36) is schematically shown in Fig. 10a with red line. In the model by Luque and Ebert (2009), the thunderstorm charge $Q$ and dipole moment $p$ increase linearly in time. Hence, it follows from Eq. (35) that the point $z_{*}$ and the wave front (Eq. 36) propagate downward. This qualitative analysis is consistent with the results of numerical simulations reported by Luque and Ebert (2009). Nevertheless, the above approach cannot predict the sharp prominence/streamer nucleating center arising in the center of the wave surface. This point requires the precise analytical analysis, because this effect can be due to any kind of plasma instability.

The sprite delay between a sprite and its causative +CGs can reach a few hundred ms (Cummer, 2003; Cummer et al., 2006a). It appears that the initiation of the long delayed sprites can be the result of the long-lasting intense CC (about hundreds of ms (Rakov and Uman, 2003) in the positive causative lightning (Reising et al., 1996; Cummer and Füllekrug, 2001; Lyons, 2006; Hu et al., 2007). The important role of higher frequency components (like Mcomponent) in the CC in explaining the long-delay sprites has been suggested (Yashunin et al., 2007; Asano et al., 2009a, b; Kudintseva et al., 2010). Ohkubo et al. (2005), Bell et al. (1998) and Cho and Rycroft $(1998,2001)$ have speculated that horizontal lightning currents between clouds and intra-cloud (IC) lightning discharges could be responsible for non-uniform ionization of the upper atmosphere and the enhancement of mesospheric electric field followed by the sprite generation. Kudintseva et al. (2010) have emphasized the relevance of transient electric field that resulted from interference between electromagnetic wave radiated by the horizontal branch of the parent lightning discharge and the waves reflected from the night ionosphere and the ground. The modeling has revealed the non-stationary fine structure in the spatial distribution of electric field in the mesosphere 
above the lightning discharge. The peak value of the electric field exceeds the runaway electron threshold that supports the idea of free electron bunching in the mesosphere by the pulsed electric field (Kudintseva et al., 2010). Based on comparison between observations and model calculations, Gamerota et al. (2011) have assumed that the sprites can be initiated in the vicinity of inhomogeneities in comparision to streamer generation as observed in laboratory tests. Luque and Gordillo-Vázquez (2012) have emphasized the role of associative detachment (Eq. 6) in time lag and altitude of the generation of delayed sprites.

The high-resolution video and telescopic imagining of the sprite filament evolution can serve as indirect evidence that overall the sprite structure is physically similar to the streamer zone observed in laboratory at atmospheric pressure (Luque and Ebert, 2009, 2010; Liu, 2010). Nevertheless, the runaway breakdown cannot be ruled out since this mechanism can trigger the streamers formation.

In conclusion we consider a possible scenario of the runaway electron breakdown in a strong QE thunderstorm field originated just after a $+\mathrm{CG}$ return stroke (Gurevich and Zybin, 2001). As we have noted above, the incident cosmic particles can serve as a source of the extensive EAS, which contains the secondary fast seed electrons (Gurevich and Zybin, 2005). The upward-propagating runaway electrons over the thunderstorm can be the result of the interaction of the secondary/seed fast electrons with massive nuclei of molecules as schematically shown in Fig. 4. The ionization of neutral molecules due to their interaction with the upwardpropagating runaway electrons gives rise to a great number of thermal electrons, which dominate over the runaway electrons. We can thus speculate about this population of thermal electrons which can serve as a nucleus for generation of the initial streamer.

It is clear from Fig. 9 that the condition $E>E_{\mathrm{c}}$ holds in the altitude range of the order of tens of $\mathrm{km}$, which is greater than the length $l_{\mathrm{a}}$ (Eq. 28) of exponential growth of runaway electron avalanche. Owing to the exponential growth of the upward-propagating electron avalanches and because of the diffusion beam broadening, the lateral dimension of the total avalanche region can reach a value about $30 \mathrm{~km}$, which is the order of the scale of the brightest sprite region at the altitude 40-60 km (Gurevich and Zybin, 2001).

In principle the runaway breakdown can explain the existence of tendrils/plasma filament in the bottom of sprite as well. To speculate about this, we shall make our own estimate of the characteristic tendril length based on this mechanism. Consider first the flux of seed fast electrons resulted from the EAS. These fast electrons cannot create downward runaway avalanches because they move against the electric force, but they can excite downward avalanches of thermal electrons followed by enhancement of the air conductivity that in turn can trigger the streamers formation, that is, the tendril-shaped structures in the bottom of sprite.
The length of these tendrils is limited by the mean distance travelled by the fast electron before it will be thermalized. To estimate this length, we consider a 1-D model in which the highly collimated beam of the fast electrons moves downwards along the downward-directed vertical axis $z$. The thunderstorm QE field $\boldsymbol{E}(z)$ is positive parallel to z-axis. In this simplest model, the fast electron undergoes the electric force $-e \boldsymbol{E}(z)$ and the dynamic friction force $\boldsymbol{F}_{\mathrm{fr}}(\varepsilon, z)$, where $\varepsilon$ is the fast electron energy. Both forces have negative projection on the axis $z$ since they decelerate the electron. Assuming that the electric field is a constant and the friction force is independent of altitude, Eqs. (18) and (19) reduce to

$\frac{d \varepsilon}{d z}=-e E-F_{\mathrm{fr}}(\varepsilon)$,

where $d \varepsilon$ is the electron energy loss within the length $d z$ due to both the electrical and friction forces. Notice that this equation follows from the principle of energy conversation.

Integrating Eq. (37) yields

$z=\int_{\varepsilon}^{\varepsilon_{\text {in }}} \frac{d \varepsilon}{e E+F_{\mathrm{fr}}(\varepsilon)}$,

where $\varepsilon_{\text {in }}=\varepsilon(0)>\varepsilon_{\mathrm{c}}$ is initial kinetic energy of the fast electron at $z=0$.

The friction force for non-relativistic electrons is given by Eq. (20). As the electron energy exceeds approximately $10 \mathrm{MeV}$, the friction force begins to increase as shown in Fig. 3 because of the relativistic effect. For simplicity, we will ignore the relativistic correction to Eq. (20). Additionally, the density of neutrals is assumed to be constant and Bethe logarithm, $\Lambda=\ln \varepsilon / J_{\mathrm{Z}}$, in Eq. (20) is considered as a constant. Substituting Eq. (20) for $F_{\text {fr }}(\varepsilon)$ into Eq. (38) and performing integration over $\varepsilon$, we arrive at

$z=\frac{1}{e E}\left(\varepsilon_{\text {in }}-\varepsilon+\varepsilon_{\mathrm{c}} \ln \frac{\varepsilon+\varepsilon_{\mathrm{c}}}{\varepsilon_{\text {in }}+\varepsilon_{\mathrm{c}}}\right)$.

To estimate the distance $z_{\max }$ required of the fast electron thermalization, one should substitute $\varepsilon=\varepsilon_{\mathrm{c}}$ into Eq. (39). The charge moment change $\Delta M_{q}$ for initiation of sprite is believed to be of the order of $500 \mathrm{C} \mathrm{km}$ (e.g. see Cummer, 2003). To estimate QE field arising just after a +CG stroke, one may substitute $\Delta M_{q} \approx 2 \times 10^{3} \mathrm{C} \mathrm{km}$ into Eq. (34) instead of dipole moment $p$. Whence, it follows that $E \approx$ $0.3 \mathrm{kV} \mathrm{m}^{-1}$ at altitude $50 \mathrm{~km}$. Substituting this value of electric field into Eq. (39) and taking the initial electron energy $\varepsilon_{\text {in }} \approx 10 \varepsilon_{\mathrm{c}} \approx 3-5 \mathrm{MeV}$ gives the value $z_{\max }=7-12 \mathrm{~km}$, which is compatible with the tendril length observed at the bottom of carrot sprites.

As we have noted above, the upward-propagating runaway avalanches and streamers can arise from the body of an earlier downward tendrils/plasma filaments followed by generation of downward and upward expanding column according to the model shown in Fig. 10b. Eventually, one may expect 
multiplication of both runaway and thermal electrons that can result in formation of the diffusion zone. It should be noted that a portion of the upward-moving fast electrons can turn their trajectories through the angle greater than $90^{\circ}$ due to strong interaction with nuclei. As a result the downwardmoving population of the fast electrons can be supported by this portion of electrons coming from the upper diffusion zone of the sprite that in turn can serve as additional source for tendril formation. So the runaway electrons can trigger both downward and upward propagating streamer.

It should be noted that the characteristic length (Eq. 28) of runaway avalanches in the upper atmosphere is so large as to present problems for laboratory tests, because it is difficult to obtain visible images of the runaway breakdown process in order to compare these laboratorial images with sprite structure observed in in-situ measurements. Nevertheless, the runaway mechanism can serve as a promising candidate for explaining TGFs (e.g. see Milikh and Roussel-Dupré, 2010).

A sophisticated treatment of runaway breakdown is based on the Boltzmann transport Eq. (25) for the distribution function of fast electrons. In a simple model, the ambient electric field, the number density of neutrals and their nucleus charge $Z e$ are assumed to be constant. It is usually the case that the solution of Eq. (25) is assumed to be independent of spatial coordinates; that is, Eq. (25) is solved only in the momentum space (p). Some numerical modelings that take into account the initial charge distribution and temporal dependence of runaway electron process have been treated in more detail (e.g. Taranenko and Roussel-Dupré, 1996; Babich et al., 1998; Symbalisty et al., 1998; Roussel-Dupré et al., 1998, 2008; Yukhimuk et al., 1998, 1999, Lehtinen et al., 1999; Milikh and Roussel-Dupré, 2010).

At first glance, the comparison between the streamer type and the runaway electron mechanism shows that the threshold for runway breakdown, shown in Fig. 9 with line 7, is approximately an order of magnitude smaller than that for the conventional breakdown. On the other hand, the focusing of the electric field in the vicinity of any inhomogeneity could lower the conventional threshold $E_{\mathrm{k}}$ for thermal breakdown by a factor of 10 or 30 (Fernsler and Rowland, 1996). Alternatively, the actual field for runaways needs to be $2-3$ times the runaway threshold value to get sufficient ionization and airglow (Rowland, 1998). Actually, both thresholds could be closer than that shown in Fig. 9 in such a way that the conventional breakdown and runaway breakdown may occur simultaneously.

This implies that there may be a hybrid model of the sprite development in the mesosphere. Roussel-Dupré and Gurevich (1996) and Yukhimuk et al. (1999) have studied the model based on combination of different mechanisms, that is, the thermal breakdown in a QE field and the runaway electron mechanism. The numerical 3-D calculations for streamer propagation including production of runaway electrons have been performed (Li et al., 2009, 2010; Chanrion and Neubert, 2010). Rowland (1998) has noted that the conventional breakdown and runaway breakdown can be triggered at different altitudes, which are separated by only a few kilometers. Once either process is triggered prior to the next one, it brings the increase of plasma density followed by fast relaxation of the electric field due to polarization. As a result, this can suppress the other process from triggering.

\subsection{VLF and ELF phenomena associated with sprites}

Since direct measurements of the sprite parameters are impossible, our knowledge of underlying mechanisms of sprite generation is mostly based on video observations of sprite morphology, optical and spectral measurements (e.g. Neubert et al., 2008). Simultaneous measurements of optical emission and ELF (extremely low frequency, $<3 \mathrm{kHz}$ )/VLF (very low frequency, $3-30 \mathrm{kHz}$ ) electromagnetic field produced by both the sprite and its causative lightning are believed to be an effective technique for detecting and improvement of the spatial and temporal resolution of the sprites (Füllekrug and Constable, 2000; Sato and Fukunishi, 2003; Hobara et al., 2006; Cummer et al., 2006a, b; Surkov et al., 2010).

The perturbation of ionospheric and mesospheric conductivity due to the sprite ionization column and sprite halos has been observed by measuring changes in the amplitude and phase of signals from distant narrowband VLF transmitters passing over a thunderstorm region (Dowden et al., 1994; Inan et al., 1995, 1996a, b, 1997, 2010; Molchanov et al., 1998; Hobara et al., 2001; Otsuyama et al., 2004; Neubert et al., 2008). The perturbations of VLF transmitter signals have been observed a few ms after the causative lightning stroke. This effect referred to as early/fast Trimpis (Inan et al., 1995) is thought to be similar to lightning-induced electron precipitation effects (LEPs). The latter is due to ionization of Dregion caused by lightning discharge and whistler-induced precipitation of radiation belt electrons. What these effects have in common is that they are long-lasting processes since the duration of the early VLF Trimpi event recoveries varies from $30 \mathrm{~s}$ to $300 \mathrm{~s}$ (Neubert et al., 2008).

To simulate early VLF event recoveries observed simultaneously with sprite discharges in the D-region, a set of continuity equations for four kinds of charged particles (that is, electrons, positive ions, negative ions, and positive cluster ions) has been treated by Glukhov et al. (1992) and Haldoupis et al. (2009). To simplify this problem, we take into account that the early VLF perturbations are characterized by abrupt signal onsets and long recoveries. In this notation, one may neglect the short-term stage of electron production due to the ionization caused by a causative lightning and its sprite and focus on a more slowly process of plasma recombination. The continuity equation for electrons can be thus written as

$\frac{d N_{\mathrm{e}}}{d t}=-\alpha_{\mathrm{d}} N_{\mathrm{e}} N_{\mathrm{i}}-\alpha_{\mathrm{d}}^{\mathrm{c}} N_{\mathrm{e}} N_{\mathrm{x}}$, 
where $N_{\mathrm{e}}, N_{\mathrm{i}}$, and $N_{\mathrm{x}}$ stand for electrons, positive ions, and positive cluster ions number densities, respectively. Here $\alpha_{\mathrm{d}}$ denotes the coefficient of dissociative recombination, while $\alpha_{\mathrm{d}}^{\mathrm{c}}$ is the effective coefficient of recombination of electrons with positive cluster ions. A majority of positive ions are constituted by $\mathrm{O}_{2}^{+}$and $\mathrm{NO}^{+}$, while the positive cluster ions are produced from positive ions via a hydration chain reaction. Notice that the interaction between electrons and negative ions due to electron attachment and detachment does not enter this simplified equation.

Taking into account that the dissociative recombination of electrons and single positive ions dominates above $80-85 \mathrm{~km}$ (Glukhov et al., 1992; Haldoupis et al., 2009) (that is, in the altitude range of interest), one may ignore the last term on the right hand side of Eq. (40). Additionally, taking notice of plasma quasi-neutrality, one should substitute $N_{\mathrm{i}} \approx N_{\mathrm{e}}$ into Eq. (40). Integrating this equation results in usual law for binary plasmas:

$$
N_{\mathrm{e}}=\frac{N_{\mathrm{e} 0}}{1+\alpha_{\mathrm{d}} N_{\mathrm{e} 0} t},
$$

where $N_{\mathrm{e} 0}$ stands for the initial electron density that arises after short-term stage of electron production.

Substituting $N_{\mathrm{e}}=N_{\mathrm{e} 0} / 2$ into Eq. (41), we obtain the estimate for plasma relaxation time $t_{\mathrm{r}} \sim\left(\alpha_{\mathrm{d}} N_{\mathrm{e} 0}\right)^{-1}$, which defines the duration of early VLF events. In the altitude range of interest, the numerical values of parameters, i.e. $\alpha_{\mathrm{d}}=10^{-7}-3 \times 10^{-7} \mathrm{~cm}^{3} \mathrm{~s}^{-1}$ (Lehtinen and Inan, 2007) and $N_{\mathrm{e} 0}=6 \times 10^{4} \mathrm{~cm}^{-3}$ (Haldoupis et al., 2009), give the estimate $t_{\mathrm{r}} \sim(1.7-5) \times 10^{2} \mathrm{~s}$ which is compatible with the observations.

To summarize, the early VLF events seem to be a byproduct of sprite-produced extra ionization in the D-region and lower ionosphere followed by the recombination of electrons and positive ions. In this picture the duration of these events is controlled by the relaxation time $t_{\mathrm{r}}$ that it takes for the recovery of plasma density and conductivity.

A new type of the so-called "early/slow" VLF perturbations associated with sprites has been recently observed. The long onset duration of these events of up to $2.5 \mathrm{~s}$ can serve as an indirect hint about a slow change in the conductivity and ionization of the lower ionosphere (e.g. Neubert et al., 2008). The experimental evidence provided by the Crete receiver together with Nançay broadband time series has shown that the early/slow VLF events can be accompanied by a few sequential CG lightning strokes and bursts of IC lightning (Neubert et al., 2008). It was hypothesized by Haldoupis et al. (2006) that the observed ionization of the lower ionosphere builds up as a result of sprite-produced electrons accelerated by sequential electromagnetic pulses radiated upwards from horizontal IC discharges. One may speculate that the electron impact upon the ionosphere can result in the generation of secondary electron avalanche thereby ionizing the ionosphere. It seems likely that the source mechanism of this effect is still an open question.
The currents flowing inside the sprite body may result in the generation of ELF electromagnetic field at a level comparable to that produced by a causative CG flash (Cummer et al., 1998, 2006a). Measurements of ELF field currents associated with sprite-producing flashes have exhibited two current peaks. The first peak corresponds to the causative lightning, whereas the second peak was found to be simultaneous in time with sprite luminosity and it appears to be proportional in amplitude to the brightness of the sprite. Based on the ELF field measurements, the charge moment change of the sprite can be extracted (Boccippio et al., 1995; Hobara et al., 2001, 2006; Cummer, 2003; Hayakawa et al., 2004; Matsudo et al., 2009). In several cases the sprite current moments responsible for the ELF pulse were estimated to be of several hundred kA km (Cummer et al., 1998, 2006a).

Interference between electromagnetic fields, $\boldsymbol{B}_{\mathrm{c}}$, originated from the causative lightning discharge, and from the delayed sprite, $\boldsymbol{B}_{\mathrm{s}}$, may result in an approximately quasioscillatory shape of ULF/ELF power spectrum of magnetic variations (Surkov et al., 2010). The spectrum of net magnetic field variations resulted from the causative lightning and the delayed sprite is given by

$\boldsymbol{B}(\omega)=\boldsymbol{B}_{\mathrm{c}}\left(\boldsymbol{r}_{\mathrm{c}}, \omega\right)+\boldsymbol{B}_{\mathrm{s}}\left(\boldsymbol{r}_{\mathrm{s}}, \omega\right) \exp (i \omega T)$,

where $\omega$ is the frequency, $T$ is the sprite time lag, $\boldsymbol{r}_{\mathrm{c}}$ and $\boldsymbol{r}_{\mathrm{s}}$ are the position vectors of the lightning and sprite, respectively. Taking into account that the power spectrum is proportional to $|\boldsymbol{B}(\omega)|^{2}$, it is obvious that the presence of the oscillatory factor $\exp (i \omega T)$ in Eq. (42) will cause the amplitude modulation of the power spectrum with "period" $\omega=2 \pi / T$. A technique of extracting sprite parameters based on the sprite-producing lightning power spectrum has been recently proposed (Surkov et al., 2010). The ULF/ELF measurements thus show considerable promise for understanding of the role played by long-lasting CC and intra-cloud processes in the delayed sprite generation.

\section{Discussion and conclusions}

The high-speed video observations and the high-resolution telescopic images of sprite formation have shown that overall the sprite structure is close to that of the streamer-like discharges observed in the laboratory (Cummer et al., 2006b; McHarg et al., 2007; Neubert et al., 2008) that are in favor of the streamer mechanism of the air breakdown in the sprites. Moreover, the shape of BJs and GJs has a close similarity with the inverted image of conventional CG discharge and thus can be interpreted in terms of the streamer-leader mechanism of the air breakdown. It is generally believed that this concept based on streamer and leader propagation at mesosphere reflects basics of the sprite and jet physics (e.g. see Pasko, 2006; Raizer et al., 2010). Nevertheless, at the moment the theory is not far enough advanced to explain the sprite behavior and the relationship between sprites and their 
causative lightning in any detail (e.g. Neubert et al., 2008; Pasco, 2010; Raizer et al., 2010). The polarity asymmetry of sprite-producing lightning (Boccippio et al., 1995; Lyons, 2006; Rycroft, 2006; Pasko, 2006; Williams et al., 2007), as well as the fact that the charge moment change of the causative $+\mathrm{CG}$ must be greater than $\sim 500 \mathrm{C} \mathrm{km}$ to produce sprites (Stanley et al., 2000; Cummer, 2003; Rycroft, 2006), is far from being well understood.

One of the challenges of sprite simulation is a variety of the sprite types and their complex structures. So the numerical modeling has been a basic instrument for theoretical study of sprite behavior except for simple estimates, which follow the similarity law. The majority of sprite simulations are based on assumption that QE field arising at mesospheric altitudes just after strong +CG lightning must exceed the threshold for the streamer propagation (e.g. see Pasko, 2010). According to the new mode of thought, the breakdown field may arise at the front of electron density/screeningionization wave originating from the lower ionosphere after $+\mathrm{CG}$ and then propagating downward in background plasma (Luque and Ebert, 2009, 2010, 2012). The computer simulations have shown that this wave can be compressed in transverse direction (Derks et al., 2008) and then be transformed into downward-propagating streamer head. This interesting result may be indicative of any kind of plasma instability and thus must be supported by an analytical condition that has not been established yet.

The models based on a combination of the streamer and runaway electron mechanisms can be useful (e.g. see Roussel-Dupré and Gurevich, 1996; Yukhimuk et al., 1999; Li et al., 2009, 2010; Chanrion and Neubert, 2010), because the runaway electron mechanism has lower breakdown threshold than the conventional one and thus it can trigger the sprite streamers as well. In this picture the air breakdown in mesosphere can start with the runaway electron avalanches followed by ionization and generation of thermal electrons that gives way to formation of the sprite streamers. Our own estimates have shown that this mechanism can explain 7-8 km length of the sprite tendrils. An observational hint that the runaway electron mechanism does occur in the atmosphere is the observations of TGFs. It appears that these events are due to Bremsstrahlung emissions from energetic $(\sim 1 \mathrm{MeV})$ electrons interacting with neutral molecules (e.g. see Dwyer et al., 2010; Carlson et al., 2010).

As we have noted above, the majority of the theoretical study deals with the numerical simulations of TLEs, which cannot provide us with analytical results/formulae. These simulations cannot explain what kind of parameters/conditions can influence upon sprite structure and branching of sprite streamers, especially taking into consideration the difference between upward and downward propagating streamers of different polarities. Moreover, the relationship between the sprite structure and meteorological conditions, IC lightning activity, and etc. does not enter these models at all. Physical mechanism of the delayed sprite is un- clear since we do not have analytical formulae related to the sprite current/charge moment change and time lag to the parameters of causative lightning. There are a lot of such problems to be solved.

To summarize, this review has demonstrated that the problem of underlining mechanism for TLEs is very complex. Despite much progress toward an understanding of the origin of TLEs, which has been recently achieved, the analytical theory of these phenomena is still far from accurate. A great deal of work has to be done to sort out this interesting problem and to distinguish between different physical mechanisms that may have a dominant role in TLE origin.

Acknowledgements. Topical Editor P. M. Ruti thanks A. P. Nickolaenko, L. F. Chernogor, and one anonymous referee for their help in evaluating this paper.

\section{References}

Allen, N. L. and Ghaffar, A.: The conditions required for propagation of cathode-directed positive streamer in air, J. Phys. D: Appl. Phys., 28, 331-337, 1995.

Asano, T., Suzuki, T., Hayakawa, M., and Cho, M. G.: Threedimensional EM computer simulation on sprite initiation above a horizontal lightning discharge, J. Atmos. Solar-terr. Phys., 71, 983-990, 2009a.

Asano, T., Suzuki, T., Hiraki, Y., Mareev, E., Cho, M. G., and Hayakawa, M.: Computer simulations on sprite initiation for realistic lightning models with higher-frequency surges, J. Geophys. Res., 114, A02310, doi:10.1029/2008JA013651, $2009 \mathrm{~b}$.

Babaeva, N. Y. and Naidis, G. V.: Dynamics of positive and negative streamers in air in weak uniform electric fields, IEEE Trans. Plasma Sci., 25, 375-379, 1997.

Babich, L. P.: High-energy phenomena in electric discharges in dense gases: Theory, experiment and natural phenomena, ISTC Science and Technology Series, Vol. 2, Futurepast, Arlington, Virginia, 2003.

Babich, L. P., Kutsyk, I. M., Donskoy, E. N., and Kudryavtsev, A. Yu.: New data on space and time scales of relativistic runaway electron avalanche for thunderstorm environment: Monte Carlo calculations, Phys. Lett. A., 245, 460-470, 1998.

Babich, L. P., Bakhov, K. I., Balakin, V. A., Donskoi, E. N., Zavada, N. I., Zelenskii, K. F., Il'kaev, R. I., Kutsyk, M., Loiko, T. V., Nedoikash, Y. M., Pavlovskaya, N. G., Roussel-Dupre, R. A., Symbalisty, E. M. D., and Shamraev, B. N.: An experimental investigation of an avalanche of relativistic runaway electrons under normal conditions, High Temp., 42, 1-11, 2004.

Babich, L. P., Kudryavtsev, A. Y., Kudryavtseva, M. L., and Kutsyk, I. M.: Atmospheric gamma-ray and neutron flashes, J. Exp. Theory Phys., 106, 65-76, 2008.

Barrington-Leigh, C. P.: Fast photometric imaging of high altitude optical flashes above thunderstorms, $\mathrm{PhD}$ dissertation, Stanford University, 2000.

Barrington-Leigh, C. P., Inan, U. S., and Stanley, M.: Identification of sprites and elves with intensified video and broadband array photometry, J. Geophys. Res., 106, A2, doi:10.1029/2000JA000073, 2001. 
Bazelyan, E. M. and Raizer, Y. P.: Spark Discharge, CRC Press, Boca Raton, 1998.

Bazilevskaya, G. A. and Svirzhevskaya, A. K.: On the stratospheric measurements of cosmic rays, Space Sci. Rev., 85, 431-521, 1988.

Bell, T. F., Reising, S. C., and Inan, U. S.: Intense continuing currents following positive cloud-cloud lightning associated with red sprites, Geophys. Res. Lett., 25, 1285-1288, 1998.

Bethe, H. A.: Zur Theorie des Durchgans schneller Korpuskularstrahlen durch Materie, Ann. Phys., Leipzig, B5, 325-400, 1930.

Boccippio, D. J., Williams, E. R., Heckman, S. J., Lyons, W. A., Baker, I. T., and Boldi, R.: Sprites, ELF transients, and positive ground strokes, Science, 269, 1088-1091, 1995.

Boeck, W. L., Vaughan Jr., O. H., Blakeslee, R., Vonnegut, B., and Brook, M.: Lightning induced brightening in the airglow layer, Geophys. Res. Lett., 19, 99-102, 1992.

Boeck, W. L., Vaughan, O. H., Blakeslee, R. J., Vonnegut, B., Brook, M., and McKune, J.: Observations of lightning in the stratosphere, J. Geophys. Res., 100, 1465-1475, 1995.

Bondiou, A. and Gallimberti, I.: Theoretical modeling of the development of the positive spark in long gaps, Journal of Physics D: Applied Physics, 27, 1252-1266, 1994.

Briggs, M. S., Fishman, G. J., Connaughton, V., Bhat, P. N., Paciesas, W. S., Preece, R. D., Wilson-Hodge, C., Chaplin, V. L., Kippen, R. M., von Kienlin, A., Meegan, C. A., Bissaldi, E., Dwyer, J. R., Smith, D. M., Holzworth, R. H., Grove, J. E., and Chekhtman, A.: First results on terrestrial gamma ray flashes from the Fermi Gamma-ray Burst Monitor, J. Geophys. Res., 115, A00E49, doi:10.1029/2009JA014853, 2010.

Carlson, B. E., Lehtinen, N. G., and Inan, U. S.: Runaway relativistic electron avalanche seeding in the Earth's atmosphere, J. Geophys. Res., 113, A10307, doi:10.1029/2008JA013210, 2008.

Carlson, B. E., Lehtinen, N. G., and Inan, U. S.: Terrestrial gamma ray flash production by active lightning leader channels, J. Geophys. Res., 115, A10324, doi:10.1029/2010JA015647, 2010.

Celestin, S. and Pasko, V. P.: Effects of spatial non-uniformity of streamer discharges on spectroscopic diagnostics of peak electric fields in transient luminous events, Geophys. Res. Lett., 37, L07804, doi:10.1029/2010GL042675, 2010.

Celestin, S. and Pasko, V. P.: Energy and fluxes of thermal runaway electrons produced by exponential growth of streamers during the stepping of lightning leaders and in transient luminous events, J. Geophys. Res., 116, A03315, doi:10.1029/2010JA016260, 2011.

Chanrion, O. and Neubert, T.: Production of runaway electrons by negative streamer discharges, J. Geophys. Res., 115, A00E32, doi:10.1029/2009JA014774, 2010.

Chen, A. B., Kuo, C.-L., Lee, Y.-J., Su, H.-T., Hsu, R.-R., Chern, J.-L., Frey, H. U., Mende, S. B., Takahashi, Y., Fukunishi, H., Chang, Y.-S., Liu, T.-Y., and Lee, L.-C.: Global distributions and occurrence rates of transient luminous events, J. Geophys. Res., 113, A08306, doi:10.1029/2008JA013101, 2008.

Cheng, Z., Cummer, S. A., Su, H. T., and Hsu, R. R.: Broadband very low frequency measurement of $\mathrm{D}$ region ionospheric perturbations caused by lightning electromagnetic pulses, J. Geophys. Res., 112, A06318, doi:10.1029/2006JA011840, 2007.

Chern, J. L., Hsu, R. R., Su, H. T., Mende, S. B., Fukunishi, H., Takahashi, Y., and Lee, L. C.: Global survey of upper atmo- spheric transient luminous events on the ROCSAT-2 satellite, J. Atmos. Sol. Terr. Phys., 65, 647-659, 2003.

Cho, M. and Rycroft, M. J.: Computer simulation of the electric field structure and optical emission from cloud-top to the ionosphere, J. Atmos. Sol.-Terr. Phys., 60, 871-888, 1998.

Cho, M. and Rycroft, M. J.: Non-uniform ionization of the upper atmosphere due to the electromagnetic pulse from a horizontal lightning discharge, J. Atmos. Solar-Terr. Phys., 63, 559-580, 2001.

Colman, J. J., Roussel-Dupré, R. A., and Triplett, L.: Temporally self-similar electron distribution functions in atmospheric breakdown: The thermal runaway regime, J. Geophys. Res., 115, A00E16, doi:10.1029/2009JA014509, 2010.

Cummer, S. A.: Current moment in sprite-producing lightning, J. Atmos. Solar-Terr. Phys., 65, 499-508, 2003.

Cummer, S. A. and Füllekrug, M.: Unusually intense CC in lightning produces delayed mesospheric breakdown, Geophys. Res. Lett., 28, 495-498, 2001.

Cummer, S. A., Inan, U. S., Bell, T. F., and Barrington-Leigh, C. P.: ELF radiation produced by electrical currents in sprites, Geophys. Res. Lett., 25, 1281-1284, 1998.

Cummer, S. A., Zhai, Y., Hu, W., Smith, D. M., Lopez, L. I., and Stanley, M. A.: Measurements and implications of the relationship between lightning and terrestrial gamma ray flashes, Geophys. Res. Lett., 32, L08811, doi:10.1029/2005GL022778, 2005.

Cummer, S. A., Frey, H. U., Mende, S. B., Hsu, R.-R., Su, H.-T., Chen, A. B., Fukunishi, H., and Takahashi, Y.: Simultaneous radio and satellite optical measurements of high-altitude sprite current and lightning continuing current, J. Geophys. Res., 111, A10315, doi:10.1029/2006JA011809, 2006a.

Cummer, S. A., Jaugey, N., Li, J., Lyons, W. A., Nelsen, T. E., and Gerken, E. A.: Submillisecond imaging of sprite development and structure, Geophys. Res. Lett., 33, L04104, doi:10.1029/2005GL024969, 2006b.

Cummer, S. A., Li, J., Han, F., Lu, G., Jaugey, N., Lyons, W. A., and Nelson, T. E.: Quantification of the troposphere-toionosphere charge transfer in a gigantic jet, Nat. Geosci., 2, 1-4, doi:10.1038/NGEO607, 2009.

Derks, G., Ebert, U., and Meulenbroek, B.: Laplacian instability of planar streamer ionization fronts - an example of pulled front analysis, J. Nonlinear Sci., 18, 551-590, 2008.

Dowden, R. L., Adams, C. D. D., Brundell, J. B., and Dowden, P. E.: Rapid onset, rapid decay (RORD), phase and amplitude perturbations of VLF subionospheric transmissions, J. Atmos. Terr. Phys., 56, 1513-1527, 1994.

Dwyer, J. R.: Source mechanisms of terrestrial gamma-ray flashes, J. Geophys. Res., 113, D10103, doi:10.1029/2007JD009248, 2008.

Dwyer, J. R., Rassul, H. K., Saleh, Z., Uman, M. A., Jerauld, J., and Plumer, J. A.: X-ray bursts produced by laboratory sparks in air, Geophys. Res. Lett., 32, L20809, doi:10.1029/2005GL024027, 2005.

Dwyer, J., Smith, D., Uman, M., Saleh, Z., Grefenstette, B., Hazelton, B., and Rassoul, H.: Estimation of the fluence of high-energy electron bursts produced by thunderclouds and the resulting radiation doses received in aircraft, J. Geophys. Res., 115, D09206, doi:10.1029/2009JD012039, 2010.

Ebert, U. and Sentman, D.: Editorial Review: Streamers, sprites, leaders, lightning: From micro- to macroscales, J. Phys. D Appl. 
Phys., 41, 230301, doi:10.1088/0022-3727/41/23/230301, 2008.

Ebert, U., Montijn, C., Briels, T. M. P., Hundsdorfer, W., Meulenbroek, B., Rocco, A., and van Veldhuizen, E. M.: The multiscale nature of streamers, Plasma Sources Sci. Technol., 15, S118S129, 2006.

Ebert, U., Nijdam, S., Li, C., Luque, A., Briels, T., and van Veldhuizen, E.: Review of recent results on streamer discharges and discussion of their relevance for sprites and lightning, J. Geophys. Res., 115, A00E43, doi:10.1029/2009JA014867, 2010.

Eletsky, A. V., Palkina, L. A., and Smirnov, B. M.: Phenomena of Transfer in Weakly Ionized Plasma, Moscow, 1975 (in Russian).

Fernsler, R. and Rowland, H.: Models of lightning-produced sprites and elves, J. Geophys. Res., 101, 29653-29662, 1996.

Fishman, G. J., Bhat, P. N., Mallozzi, R., Horack, J. M., Koshut, T., Kouveliotou, C., Pendleton, G. N., Meegan, C. A., Wilson, R. B., Paciesas, W. S., Goodman, S. J., and Christian, H. J.: Discovery of intense gamma-ray flashes of atmospheric origin, Science, 264, 1313-1316, 1994.

Franz, R. C., Nemzak, R. J., and Winkler, J. R.: Television image of a large upward electrical discharge above a thunderstorm system, Science, 249, 48-51, 1990.

Fukunishi, H., Takahashi, Y., Fujito, M., Wanatabe, Y., and Sakanoi, S.: Fast imagining of elves and sprites using a framing/streak camera and a multi-anode array photometer, EOS Trans. AGU Fall Meet. Suppl., 77, F60, 1996.

Füllekrug, M. and Constable, S.: Global triangulation of intense lightning discharges, Geophys. Res. Lett., 27, 333-336, 2000.

Füllekrug, M., Roussel-Dupré, R. A., Symbalisty, E. M. D., Chanrion, O., Odzimek, A., der Velde, O. V., and Neubert, T.: Relativistic runaway breakdown in low-frequency radio, J. Geophys. Res., 115, A00E09, doi:10.1029/2009JA014468, 2010.

Füllekrug, M., Roussel-Dupré, R., Symbalisty, E. M. D., Colman, J. J., Chanrion, O., Soula, S., van der Velde, O., Odzimek, A., Bennett, A. J., Pasko, V. P., and Neubert, T.: Relativistic electron beams above thunderclouds, Atmos. Chem. Phys., 11, 77477754, doi:10.5194/acp-11-7747-2011, 2011.

Gamerota, W. R., Cummer, S. A., Li, J., Stenbaek-Nielsen, H. C., Haaland, R. K., and McHarg, M. G.: Comparison of sprite initiation altitudes between observation and models, J. Geophys. Res., 116, A02317, doi:10.1029/2010JA016095, 2011.

Glukhov, V., Pasko, V., and Inan, U.: Relaxation of transient lower ionospheric disturbances caused by lightning-whistler-induced electron precipitation bursts, J. Geophys. Res., 97, 16971-16979, 1992.

Gurevich, A. V. and Zybin, K. P.: Kinetic equation for high energy electrons in gases, Phys. Lett. A, 237, 240-246, 1998.

Gurevich, A. V. and Zybin, K. P.: Runaway breakdown and electric discharges in thunderstorm, Phys.-Uspekhi, 44, 1119-1140, 2001

Gurevich, A. V. and Zybin, K. P.: High energy cosmic ray particles and the most powerful discharges in thunderstorm atmosphere, Phys. Lett., A329, 341-347, doi:10.1016/j.physleta.2004.06.094, 2004.

Gurevich, A. V. and Zybin, K. P.: Runaway breakdown and the mysteries of lightning, Physics Today, May, 37-43, 2005.

Gurevich, A. V., Milikh, G. M., and Roussel-Dupré, R.: Runaway electron mechanism of air breakdown and preconditioning during a thunderstorm, Phys. Lett. A, 165, 463-468, 1992.
Gurevich, A. V., Milikh, G. M., and Roussel-Dupré, R.: Nonuniform runaway air-breakdown, Phys. Lett. A, 187, 197-203, 1994

Gurevich, A. V., Valdivia, J. A., Milikh, G. M., and Papadopoulos, K.: Runaway electrons in the atmosphere in the presence of magnetic field, Radio Sci., 31, 1541-1554, 1996.

Gurevich, A. V., Sergeichev, K. F., Sychov, I. A., Roussel-Dupré, R., and Zybin, K. P.: First observations of runaway breakdown phenomenon in laboratory experiments, Phys. Lett. A, 260, 269272, 1999a.

Gurevich, A. V., Zybin, K. P., and Roussel-Dupré, R. A.: Lightning initiation by simultaneous effect of runway breakdown and cosmic ray shower, Phys. Lett. A., 254, 79-97, 1999b.

Gurevich, A. V., Carlson, H. C., Medvedev, Yu. V., and Zybin, K. P.: Generation of electron-positron pairs in runaway breakdown, Phys. Lett. A, 275, 101-108, 2000.

Gurevich, A. V., Karashtin, A. N., Chubenko, A. P., Duncan, L. M., Ryabov, V. A., Shepetov, A. S., Antonova, V. P., Kryukov, S. V., Piscal, V. V., Ptitsyn, M. O., Vildanova, L. I., Shlyugaev, Yu. V., and Zybin, K. P.: Experimental evidence of giant electron - gamma bursts generated by extensive atmospheric shower in thunderclouds, Phys. Lett., A325, 389-402, doi:10.1016/j.physleta.2004.03.074, 2004.

Gurevich, A., Zybin, K., and Medvedev, Y.: Runaway breakdown in strong electric field as a source of terrestrial gamma flashes and gamma bursts in lightning leader steps, Phys. Lett., A361, 119-125, 2007.

Gurevich, A. V., Karashtin, A. N., Ryabov, V. A., Chubenko, A. P., and Shchepetov, S. V.: Nonlinear phenomena in the ionospheric plasma. Effects of cosmic rays and runaway breakdown on thunderstorm discharges, Phys. Uspekhi, 52, 735-745, doi:10.3367/UFN0179.200907h.0779, 2009.

Haldoupis, C., Steiner, R. J., Mika, Á., Shalimov, S., Marshall, R. A., Inan, U. S., Bösinger, T., and Neubert, T. "Early/slow" events: a new category of VLF perturbations observed in relation with sprites, J. Geophys. Res., 111, A11321, doi:10.1029/2006JA011960, 2006.

Haldoupis, C., Mika, Á., and Shalimov, S.: Modeling the relaxation of early VLF perturbations associated with transient luminous events, J. Geophys. Res., 114, A00E04, doi:10.1029/2009JA014313, 2009.

Hayakawa, M., Nakamura, T., Hobara, Y., and Williams, E.: Observation of sprites over the Sea of Japan and conditions for lightning-induced sprites in winter, J. Geophys. Res., 105, 46894697, 2004.

Heavner, M. J., Sentman, D. D., Moudry, D. R., and Wescott, E. M.: Sprites, blue jets, and elves: Optical evidence of energy transport across the stratopause, in: Atmospheric Science Across the Stratosphere, edited by: Siskind, D. E., Eckermann, S. D., and Summers, M. E., Geophysical Monograph Series, AGU, vol. 123, 69-82, 2000.

Hiraki, Y. and Fukunishi, H.: Theoretical criterion of charge moment change by lightning for initiation of sprites, J. Geophys Res., 111, A11305, doi:10.1029/2006JA011729, 2006.

Hobara, Y., Iwasaki, N., Hayashida, T., Hayakawa, M., Ohta, K., and Fukunishi, H.: Interrelation between ELF transients and ionospheric disturbances in association with sprites and elves, Geophys. Res. Lett., 28, 935-938, 2001.

Hobara, Y., Hayakawa, M., Williams, E., Boldi, R., and Downes, E.: Location and electrical properties of sprite-producing light- 
ning from a single ELF site, in: Sprites, Elves and Intense Lightning Discharges, NATO Sci. Ser., Ser. II, vol. 225, edited by: Füllekrug, M., Mareev, E. A., and Rycroft, M. J., pp. 211-235, Springer, Dordrecht, Netherlands, 2006.

$\mathrm{Hu}, \mathrm{W}$., Cummer, S. A., and Lyons, W. A.: Testing sprite initiation theory using lightning measurements and modeled EM fields, J. Geophys. Res., 112, D13115, doi:10.1029/2006JD007939, 2007.

Huxley, G. H. and Crompton, R. W.: The Diffusion and Drift of Electrons in Gases, John Wiley \& Sons Inc., New York, 1974.

Inan, U. S. and Lehtinen, N. G.: Production of terrestrial gammaray flashes by an electromagnetic pulse from a lightning return stroke, Geophys. Res. Lett., 32, L19818, doi:10.1029/2005GL023702, 2005.

Inan, U. S., Bell, T. F., Pasko, V. P., Sentman, D. D., Wescott, E. M., and Lyons, W. A.: VLF signatures of ionospheric disturbances associated with sprites, Geophys. Res. Lett., 22, 34613464, doi:10.1029/95GL03507, 1995.

Inan, U. S., Reising, S. C., Fishman, G. J., and Horack, J. M.: On the association of terrestrial gamma-ray bursts with lightning and implications for sprites, Geophys. Res. Lett., 23, 1017-1020, 1996a.

Inan, U. S., Sampson, W. A., and Taranenko, Y. N.: Spacetime structure of optical flashes and ionization changes produced by lightning-EMP, Geophys. Res. Lett., 23, 133-136, doi:10.1029/95GL03816, 1996b.

Inan, U. S., Barrington-Leigh, C., Hansen, S., Glukhov, V. S., Bell, T. F., and Rairden, R.: Rapid lateral expansion of optical luminosity in lightning-induced ionospheric flashes referred to as "elves", Geophys. Res. Lett., 24, 583-586, 1997.

Inan, U. S., Cummer, S. A., and Marshall, R. A.: A survey of ELF and VLF research on lightning-ionosphere interactions and causative discharges, J. Geophys. Res., 115, A00E36, doi:10.1029/2009JA014775, 2010.

Krehbiel, P. R., Riousset, J. A., Pasko, V. P., Thomas, R. J., Rison, W., Stanley, M. A., and Edens, H. E.: Upward electrical discharges from thunderstorms, Nat. Geosci., 1, 233-237, doi:10.1038/ngeo162, 2008.

Kudintseva, I. G., Nickolaenko, A. P., and Hayakawa, M.: Transient electric field in the mesosphere above a $\Gamma$-shape lightning stroke, Surv. Geophys., 31, 427-448, doi:10.1007/s10712-010-9095-x, 2010.

Kuo, C., Chen, A. B., Lee, Y. J., Tsai, L. Y., Chou, R. K., Hsu, R. R., Su, H. T., Lee, L. C., Cummer, S. A., Frey, H. U., Mende, S. B., Takahashi, Y., and Fukunishi, H.: Modeling elves observed by FORMOSAT-2 satellite, J. Geophys. Res., 112, A11312, doi:10.1029/2007JA012407, 2007.

Kuo, C., Chou, J. K., Tsai, L. Y., Chen, A. B., Su, H. T., Hsu, R. R., Cummer, S. A., Frey, H. U., Mende, S. B., Takahashi, Y., and Lee, L. C.: Discharge process, electric field, and electron energy in ISUAL-recorded gigantic jets, J. Geophys. Res., 114, A04314, doi:10.1029/2008JA013791, 2009.

Kurzan, B., Steuer, K.-H., and Fussman, G.: Dynamics of runaway electrons in the magnetic field of a tokamak, Phys. Rev. lett., 75, 4626-4629, 1995.

Kuznetsov, Yu. K., Chamaa Neto, I. El., Nascimento, I. C., and Galvão, R. M. O.: Runaway discharge in the small Brazilian Tokamak TBR-1, Phys. Plasmas, 6, 4002-4007, doi:10.1063/1.873662, 1999.
Landau, L. D. and Lifshits, E. M.: Electrodynamics of Continuous Media, Nauka, Moscow, 1982.

Lehtinen, N.: Relativistic runaway electrons above thunderstorms, Ph.D. dissertation, Stanford University, 2000.

Lehtinen, N. G. and Inan, U. S.: Possible persistent ionization caused by giant blue jets, Geophys. Res. Lett., 34, L08804, doi:10.1029/2006GL029051, 2007.

Lehtinen, N., Bell, T., Pasko, V., and Inan, U.: A two-dimensional model of runaway electron beams driven by quasi-electrostatic thundercloud fields, Geophys. Res. Lett., 24, 2639-2642, 1997.

Lehtinen, N., Bell, T., and Inan, U.: Monte Carlo simulation of runaway $\mathrm{MeV}$ electron breakdown with application to red sprites and terrestrial gamma ray flashes, J. Geophys. Res., 104, 2469924712, 1999.

Lehtinen, N. G., Inan, U. S., and Bell, T. F.: Effects of thunderstormdriven runaway electrons in the conjugate hemisphere: Purple sprites, ionization enhancements, and gamma rays, J. Geophys. Res., 106, 28841-28856, 2001.

Li, J. and Cummer, S. A.: Measurement of sprite streamer acceleration and deceleration, Geophys Res. Lett., 36, L10812, doi:10.1029/2009GL037581, 2009.

Li, C., Ebert, U., and Hundsdorfer, W.: 3D hybrid computations for streamer discharges and production of run-away electrons, J. Phys. D: Appl. Phys., 42, 202003, doi:10.1088/00223727/42/20/202003, 2009.

Li, C., Ebert, U., and Hundsdorfer, W.: Spatially hybrid computations for streamer discharges with generic features of pulled fronts: I. Planar fronts, J. Comput. Phys., 229, 200-220, 2010.

Lieberman, M. A. and Lichtenberg, A. J.: Principles of Plasma Discharges and Material Processing, John Wiley \& Sons Inc., 1994.

Liu, N.: Model of sprite luminous trail caused by increasing streamer current, Geophys. Res. Lett., 37, L04102, doi:10.1029/2009GL042214, 2010.

Liu, N. Y. and Pasko, V. P.: Effects of photoionization on similarity properties of streamers at various pressures in air, J. Phys. D Appl. Phys., 39, 327-334, doi:10.1088/0022-3727/39/2/013, 2006.

Liu, N. Y., Pasko, V. P., Adams, K., Stenbaek-Nielsen, H. C., and McHarg, M. G.: Comparison of acceleration, expansion, and brightness of sprite streamers obtained from modeling and high-speed video observations, J. Geophys. Res., 114, A00E03, doi:10.1029/2008JA013720, 2009.

Luque, A. and Ebert, U.: Emergence of sprite streamers from screening-ionization waves in the lower ionosphere, Nature Geosci., 2, 2, doi:10.1038/ngeo662, 2009.

Luque, A. and Ebert, U.: Sprites in varying air density: Charge conservation, glowing negative trails and changing velocity, Geophys. Res. Lett., 37, L06806, doi:10.1029/2009GL041982, 2010.

Luque, A. and Ebert, U.: Density models for streamer discharges: beyond cylindrical symmetry and homogeneous media, J. Comput. Phys., 231, 904-918, doi:10.1016/j.jcp.2011.04.019, 2012.

Luque, A. and Gordillo-Vázquez, F. J.: Sprite beads originating from inhomogeneities in the mesospheric electron density, Geophys. Res. Lett., 38, L04808, doi:10.1029/2010GL046403, 2011.

Luque, A. and Gordillo-Vázquez, F. J.: Mesospheric electric breakdown and delayed sprite ignition caused by electron detachment, Nature Geosci., 5, doi:10.1038/ngeo1357, 2012.

Lyons, W. A.: The meteorology of transient luminous events. An introduction and overview, in: Sprites and Intense Lightning Dis- 
charges, NATO Sci. Ser., Ser. II, vol. 225, edited by: Füllekrug, M., Mareev, E. A., and Rycroft, M. J., pp. 19-44, Springer, Dordrecht, Netherlands, 2006.

Lyons, W. A., Nelson, T., Williams, E. R., Cummer, S. A., and Stanley, M. A.: Characteristics of sprite-producing positive cloud-toground lightning during the 19 July STEPS mesoscale convective systems, Mon. Weather Rev., 131, 2417-2427, 2003.

Mareev, E. A. and Trakhtengerts, V. Y.: Puzzles of atmospheric electricity, Priroda, 3, 24-33, 2007 (in Russian).

Marshall, T., McCarthy, M. P., and Rust, W. D.: Electric field magnitudes and lightning initiation in thunderstorms, J. Geophys. Res., 100, 7097-7103, doi:10.1029/95JD00020, 1995.

Matsudo, Yu., Suzuki, T., Hayakawa, M., Yamashita, K., Ando, Y., Michimoto, K., and Korepanov, V.: Characteristics of Japanese winter sprites and their parent lightning as estimated by VHF lightning and ELF transients, J. Atmos. Solar-terr. Phys., 69, 1431-1446, 2007.

Matsudo, Y., Suzuki, T., Michimoto, K., Myokei, K., and Hayakawa, M.: Comparison of time delays of sprites induced by winter lightning flashes in the Japan Sea with those in the Pacific Ocean, J. Atmos. Solar-terr. Phys., 71, 101-111, 2009.

McHarg, M. G., Stenbaek-Nielsen, H. C., and Kanmae, T.: Observation of streamer formation in sprites, Geophys. Res. Lett., 34, L06804, doi:10.1029/2006GL027854, 2007.

Mende, S. B., Frey, H. U., Hsu, R. R., Su, H. T., Chen, A. B., Lee, L. C., Sentman, D. D., Takahashi, Y., and Fukunishi, H.: D region ionization by lightning-induced electromagnetic pulses, J. Geophys. Res., 110, 11312, doi:10.1029/2005JA011064, 2005.

Milikh, G. and Roussel-Dupré, R.: Runaway breakdown and electrical discharges in thunderstorms, J. Geophys. Res., 115, A00E60, doi:10.1029/2009JA014818, 2010.

Milikh, G. M., Valdivia, J. A., and Papadopoulos, K.: Model of red sprites optical spectra, Geophys. Res. Lett., 24, 833-836, 1997.

Mishin, E. V. and Milikh, G. M.: Blue jets: Upward lightning, Space Sci. Rev., 137, 473-488, 2008.

Molchanov, O. A., Shvets, A. V., and Hayakawa, M.: Analysis of lightning-induced ionization from VLF Trimpi events, J. Geophys. Res., 103, 23443-23458, 1998.

Montanyá, J., van der Velde, O., Romero, D., March, V., Solá, G., Pineda, N., Arrayas, M., Trueba, J. L., Reglero, V., and Soula, S.: High-speed intensified video recordings of sprites and elves over the western Mediterranean Sea during winter thunderstorms, J. Geophys. Res., 115, A00E18, doi:10.1029/2009JA014508, 2010.

Moruzzi, J. L. and Price, D. A.: Ionization, attachment and detachment in air and air-CO $\mathrm{CO}_{2}$ mixtures, J. Phys., D7, 1434-1440, 1974.

Moudry, D. R., Stenbaek-Nielsen, H. C., Sentman, D. D., and Wescott, E. M.: Imaging of elves, halos and sprite initiation at $1 \mathrm{~ms}$ time resolution, J. Atmos. Solar-Terr. Phys., 65, 509-518, doi:10.1016/S1364-6826(02)00323-1, 2003.

Myokei, K., Matsudo, Y., Asano, T., Suzuki, T., Hobara, Y., Michimoto, K., and Hayakawa, M.: A study of the morphology of winter sprites in the Hokuriku area of Japan in relation to cloud height, J. Atmos. Solar-Terr. Phys., 71, 579-602, 2009.

Nagano, M. and Watson, A. A.: Observations and implications of the ultrahigh-energy cosmic rays, Rev. Mod. Phys., 72, 689-732, 2000.

Neubert, T., Rycroft, M., Farges, T., Blanc, E., Chanrion, O., Arnone, E., Odzimek, A., Arnold, N., Enell, C.-F., Turunen, E., Bösinger, T., Mika, Á., Haldoupis, C., Steiner, R. J., van der Velde, O., Soula, S., Berg, P., Boberg, F., Thejll, T., Cristiansen, B., Ignaccolo, M., Füllekrug, M., Verronen, P. T., Montanyá, J., and Crosby, N.: Recent results from studies of electric discharges in the mesosphere, Surv. Geophys., 29, 71-137, doi:10.1007/s10712-008-9043-1, 2008.

Neubert, T., Chanrion, O., Arnone, E., Zanotti, F., Cummer, S., Li, J., Füllekrug, M., Soula, S., and van der Velde, O.: The properties of a gigantic jet reflected in a simultaneous sprite: Observations interpreted by a model, J. Geophys. Res., 116, A12329, doi:10.1029/2011JA016928, 2011.

Nickolaenko, A. P. and Hayakawa, M.: Heating of the lower ionosphere electrons by electromagnetic radiation of lightning discharges, Geophys. Res. Lett., 22, 3015-3018, 1995.

Ohkubo, A., Fukunishi, H., Takahashi, Y., and Adachi, T.: VLF/ELF sferic evidence for in-cloud discharge activity producing sprites, Geophys. Res.Lett., 32, L04812, doi:10.1029/2004GL021943, 2005.

Otsuyama, T., Manaba, J., Hayakawa, M., and Nishimura, M.: Characteristics of subionospheric VLF perturbation associated with winter lightning around Japan, Geophys. Res. Lett., 31, L04117, doi:10.1029/2003GL019064, 2004.

Paiva, G. S., Pavão, A. C., and Bastos, C. C.: "Seed" electrons from muon decay for runaway mechanism in the terrestrial gamma ray flash production, J. Geophys. Res., 114, D03205, doi:10.1029/2008JD010468, 2009.

Pancheshnyi, S.: Role of electronegative gas admixtures in streamer start, propagation and branching phenomena, Plasma Sources Science and Technology, 14, 645-653, 2005.

Papadopoulos, K., Milikh, G., Gurevich, A., Drobot, A., and Shanny, R.: Ionization rates for atmospheric and ionospheric breakdown, J. Geophys. Res., 98, 17593-17596, 1993.

Papadopoulos, K., Milikh, G., and Valdivia, J.: Comment on "Can gamma radiation be produced in the electrical environment above thunderstorms", Geophys. Res. Lett., 23, 2283-2284, 1996.

Pasko, V. P.: Theoretical modeling of Sprites and Jets, in: Sprites, Elves and Intense Lightning Discharges, NATO Sci. Ser., Ser. II, vol. 225, edited by: Füllekrug, M., Mareev, E. A., and Rycroft, M. J., pp. 255-311, Springer, Dordrecht, Netherlands, 2006.

Pasko, V. P.: Red sprite discharges in the atmosphere at high altitude: The molecular physics and the similarity with laboratory discharges, Plasma Sources Sci. Technol., 16, S13-S29, doi:10.1088/0963-0252/16/1/S02, 2007.

Pasko, V. P.: Recent advances in theory of transient luminous events, J. Geophys. Res., 115, A00E35, doi:10.1029/2009JA014860, 2010.

Pasko, V. P. and George, J. J.: Thee-dimensional modeling of blue jets and blue starters, J. Geophys. Res., 107, doi:10.1029/2002JA009473, 2002.

Pasko, V., Inan, U., Taranenko, Y., and Bell, T.: Heating, ionization and upward discharges in the mesosphere due to intense quasielectrostatic thundercloud fields, Geophys. Res. Lett., 22, 365368, 1995.

Pasko, V., Inan, U., and Bell, T.: Blue jets produced by quasielectrostatic pre-discharge thundercloud fields, Geophys. Res. Lett., 23, 301-304, 1996a.

Pasko, V., Inan, U., and Bell, T.: Sprites as luminous columns of ionization produced by quasi-electrostatic thundercloud fields, Geophys. Res. Lett., 23, 649-652, 1996 b. 
Pasko, V., Inan, U., and Bell, T.: Sprites as evidence of vertical gravity wave structures above mesoscale thunderstorms, Geophys. Res. Lett., 24, 1735-1738, 1997a.

Pasko, V., Inan, U., Bell, T., and Taranenko, Y.: Sprites produced by quasi-electrostatic heating and ionization in the lower ionosphere, J. Geophys. Res., 102, 4529-4561, 1997b.

Pasko, V., Inan, U., and Bell, T.: Spatial structure of sprites, Geophys. Res. Lett., 25, 2123-2126, 1998a.

Pasko, V., Inan, U., and Bell, T.: Mechanism of ELF radiation from sprites, Geophys. Res. Lett., 25, 3493-3496, 1998b.

Pasko, V. P., Inan, U. S., and Bell, T. F.: Fractal structure of sprites, Geophys. Res. Lett., 27, 497-500, 2000.

Pasko, V. P., Inan, U. S., and Bell, T. F.: Mesosphere-ionosphere coupling due to sprites, Geophys. Res. Lett., 28, 3821-3824, 2001.

Pasko, V. P., Stanley, M. A., Matheus, J. D., Inan, U. S., and Wood, T. G.: Electrical discharge from a thunderstorm top to the lower ionosphere, Nature, 416, 152-154, 2002.

Petrov, N. I. and Petrova, G. N.: Physical mechanism for the development of lightning discharges between a thundercloud and the ionosphere, Tech. Phys., 44, 472-475, 1999.

Rahman, M., Corray, V., Ahmad, N. A., Nyberg, J., Rakov, V. A., and Sharma, S.: X rays from $80 \mathrm{~cm}$ long sparks in air, Geophys. Res. Lett., 35, L06805, doi:10.1029/2007GL032678, 2008.

Raizer, Y. P.: Gas Discharge Physics, Springer-Verlag, Berlin Heidelberg, 1991.

Raizer, Y. P., Milikh, G. M., Shneider, M. N., and Novakovski, S. V.: Long streamer in the upper atmosphere above thundercloud, J. Phys. D.: Appl. Phys., 31, 3255-3264, 1998.

Raizer, Y. P., Milikh, G. M., and Shneider, M. N.: On the mechanism of blue jet formation and propagation, Geophys. Res. Lett., 33, L23801, doi:10.1029/2006GL027697, 2006.

Raizer, Y. P., Milikh, G. M., and Shneider, M. N.: Leader-streamers nature of blue jets, J. Atmos. Sol. Terr. Phys., 69, 925-938, doi:10.1016/j.jastp.2007.02.007, 2007.

Raizer, Y. P., Milikh, G. M., and Shneider, M. N.: Streamerand leader-like processes in the upper atmosphere: Models of red sprites and blue jets, J. Geophys. Res., 115, A00E42, doi:10.1029/2009JA014645, 2010.

Rakov, V. A.: Positive and bipolar lightning discharges: A review, Proc. 25th Int. Conf. Lightning Protection, Rhodos, pp. 103-108, 2000.

Rakov, V. A. and Uman, M. A.: Lightning: Physics and Effects, 687 pp., Cambridge Univ. Press, Cambridge, UK, 2003.

Rayment, S. W. and Moruzzi, J. L.: Electron detachment studies between $\mathrm{O}^{-}$ions and nitrogen, Int. J. Mass Spectrom. Ion Phys., 26, 321-326, 1978.

Reising, S. C., Inan, U. S., Bell, T. F., and Lyons, W. A.: Evidence for CCs in sprite-producing cloud-to-ground lightning, Geophys. Res. Lett., 23, 3639-3642, 1996.

Riousset, J. A., Pasko, V. P., and Bourdon, A.: Air-densitydependent model for analysis of air heating associated with streamers, leaders, and transient luminous events, J. Geophys. Res., 115, A12321, doi:10.1029/2010JA015918, 2010a.

Riousset, J. A., Pasko, V. P., Krehbiel, P. R., Rison, W., and Stanley, M. A.: Modeling of thundercloud screening charges: Implications for blue and gigantic jets, J. Geophys. Res., 115, A00E10, doi:10.1029/2009JA014286, 2010b.
Roussel-Dupré, R. and Gurevich, A.: On runaway breakdown and upward propagating discharges, J. Geophys. Res., 101, 22972311, 1996.

Roussel-Dupré, R. A., Gurevich, A. V., Tunnell, T., and Milikh, G. M.: Kinetic theory of runaway breakdown, Phys. Rev. E, 49, 2257-2271, 1994.

Roussel-Dupré, R., Symbalisty, E., Taranenko, Y., and Yukhimuk, V.: Simulations of high-altitude discharges initiated by runaway breakdown, J. Atmos. Solar-Terr. Phys., 60, 917-940, 1998.

Roussel-Dupré, R., Colman, J. J., Symbalisty, E., Sentman, D., and Pasko, V. P.: Physical processes related to discharges in planetary atmospheres, Space Sci. Rev., 137, 51-82, doi:10.1007/s11214008-9385-5, 2008.

Rowland, H. L.: Theories and simulations of elves, sprites and blue jets, J. Atmos. Solar-Terr. Phys., 60, 831-844, 1998.

Rycroft, M. J.: Introduction to the physics of sprites, elves and intense lightning discharges, in: Sprites, Elves and Intense Lightning Discharges, NATO Sci. Ser., Ser. II, vol. 225, edited by: Füllekrug, M., Mareev, E. A., and Rycroft, M. J., pp. 1-18, Springer, Dordrecht, Netherlands, 2006.

Sato, M. and Fukunishi, H.: Global sprite occurrence locations and rates derived from triangulation of transient Schumann resonances events, Geophys. Res. Lett., 30, 1859, doi:10.1029/2003GL017291, 2003.

Sentman, D. D. and Wescott, E. M.: Observations of upper atmosphere optical flashes recorded from an aircraft, Geophys. Res. Lett., 20, 2857-2860, 1993.

Sentman, D. D., Wescott, E. M., Osborne, D. L., Hampton, D. L., and Heavner, M. J.: Preliminary results from the Sprites 94 campaign: Red Sprites, Geophys. Res. Lett., 22, 1205-1208, 1995.

Smith, D. M., Lopez, L. I., Lin, R. P., and Barrington-Leigh, C. P.: Terrestrial gamma-ray flashes observed up to $20 \mathrm{MeV}$, Science, 307, 1085-1088, 2005.

Smith, D. M., Hazelton, B. J., Grefenstette, B. W., Dwyer, J. R., Holzworth, R. H., and Lay, E. H.: Terrestrial gamma ray flashes correlated to storm phase and tropopause height, J. Geophys. Res., 115, A00E49, doi:10.1029/2009JA014853, 2010.

Soloviev, S. P. and Surkov, V. V.: Electrostatic field and lightning generated in the gaseous dust cloud of explosive products, Geomagnetism and Aeronomy, 40, 61-69, 2000.

Stanley, M., Krehbiel, P., Brook, M., Moore, C., Rison, W., and Abrahams, B.: High speed video of initial sprite development, Geophys. Res. Lett., 26, 3201-3204, 1999.

Stanley, M., Brook, M., Krehbiel, P., and Cummer, S.: Detection of daytime sprites via a unique sprite ELF signature, Geophys. Res. Lett., 27, 871-874, 2000.

Stenbaek-Nielsen, H. C. and McHarg, M. G.: High time-resolution sprite imagining: observation and implications, J. Phys. D Appl. Phys., 41, 234009, doi:10.1088/0022-3727/41/23/234009, 2008.

Stenbaek-Nielsen, H. C., Moudry, D. R., Wescott, E. M., Sentman, D. D., and Sao Sabbas, F. T.: Sprites and possible mesospheric effects, Geophys. Res. Lett., 27, 38727-3832, 2000.

Stenbaek-Nielsen, H. C., McHarg, M. G., Kanmae, T., and Sentman, D. D.: Observed emission rates in sprite streamer heads, Geophys. Res. Lett., 34, L11105, doi:10.1029/2007GL029881, 2007.

Su, H. T., Hsu, R. R., Chen, A. B., Wang, Y. C., Hsiao, W. S., Lai, W. C., Lee, L. C., Sato, M., and Fukunishi, H.: Gigantic jets between a thundercloud and the ionosphere, Nature, 423, 974-976, 2003. 
Sukhorukov, A. I., Mishin, E. V., Stubbe, P., and Rycroft, M. J.: On blue jet dynamics, Geophys. Res. Lett., 23, 1625-1628, 1996 a.

Sukhorukov, A. I., Rudenchik, E. A., and Stubbe, P.: Simulation of the strong lightning pulse penetration into the lower ionosphere, Geophys. Res. Lett., 23, 2911-2914, 1996 b.

Surkov, V. V., Matsudo, Y., Hayakawa, M., and Goncharov, S. V.: Estimation of lightning and sprite parameters based on observation of sprite-producing lightning power spectra, J. Atmos. Sol.Terr. Phys., 72, 448-456, 2010.

Suzuki, T., Matsudo, Y., Asano, T., Hayakawa, M., and Michimoto, K.: Meteorological and electrical aspects of several winter thunderstorms with sprites in the Hokuriku area of Japan, J. Geophys. Res., 116, D06205, doi:10.1029/2009JD013358, 2011.

Symbalisty, E. M. D., Roussel-Dupré, R. A., and Yukhimuk, V. A.: Finite volume solution of the relativistic Boltzmann equation for electron avalanche studies, IEEE Trans. Plasma Sci., 26, 15751582, 1998.

Taranenko, Y. N. and Roussel-Dupré, R.: High altitude discharges and gamma ray flashes: a manifestation of runaway air breakdown, Geophys. Res. Lett., 23, 571-574, 1996.

Taylor, M. J., Bailey, M. A., Pautet, P. D., Cummer, S. A., Jaugey, N., Thomas, J. N., Solorzano, N. N., Sao Sabbas, F., Holzworth, R. H., Pinto, O., and Schuch, N. J.: Rare measurements of a sprite with halo event driven by a negative lightning discharge over Argentina, Geophys. Res. Lett., 35, L14812, doi:10.1029/2008GL033984, 2008.

Trakhtengerts, V. Y., Iudin, D. I., Kulchitsky, A. V., and Hayakawa, M.: Kinetics of runaway electrons in a stochastic electric field, Phys. Plasmas, 9, 2762-2766, 2002.

Trakhtengerts, V. Y., Iudin, D. I., Kulchitsky, A. V., and Hayakawa, M.: Electron acceleration by a stochastic electric field on the atmospheric layer, Phys. Plasma, 10, 3290-3296, 2003.

Uman, M. A.: The Lightning Discharge, Academic Press, Orlando, 1987.

Vallance-Jones, A.: Aurora, D. Reidel, Dordrecht, The Netherlands, 1974.

van der Velde, O. A., Mika, A., Soula, S., Haldoupis, C., Neubert, T., and Inan, U. S.: Observations of the relationship between sprite morphology and in-cloud lightning processes, J. Geophys. Res., 111, D15203, doi:10.1029/2005JD006879, 2006.

van der Velde, O. A., Lyons, W. A., Nelson, T. E., Cummer, S. A., Li, J., and Bunnell, J.: Analysis of the first gigantic jet recorded over continental North America, J. Geophys. Res., 112, D20104, doi:10.1029/2007JD008575, 2007.

van der Velde, O. A., Bór, J., Li, J., Cummer, S. A., Arnone, E., Zanotti, F., Füllekrug, M., Haldoupis, C., NaitAmor, S., and Farges, T.: Multi-instrumental observations of a positive gigantic jet produced by a winter thunderstorm in Europe, J. Geophys. Res., 115, D24301, doi:10.1029/2010JD014442, 2010.
Veronis, G., Pasko, V. P., and Inan, U. S.: Characteristics of mesospheric optical emissions produced by lightning discharges, J. Geophys. Res., 104, 12645-12656, 1999.

Vitello, P. A., Penetrante, B. M., and Barsley, J. N.: Simulation of negative-streamer dynamics in nitrogen, Phys. Rev. E, 49, 55745598, doi:10.1103/PhysRevE.49.5574, 1994.

Wescott, E. M., Sentman, D., Osborne, D., Hampton, D., and Heavner, M.: Preliminary results from the Sprites94 aircraft campaign: 2. Blue jets, Geophys. Res. Lett., 22, 1209-1212, 1995.

Wescott, E. M., Sentman, D. D., Heavner, M. J., Hampton, D. L., Osborne, D. L. D., and Vaugham Jr., O. H.: Blue starters: Brief upward discharges from an intense Arkansas thunderstorm, Geophys. Res. Lett., 23, 2153-2156, 1996.

Wescott, E. M., Sentman, D. D., Heavner, M. J., Hampton, D. L., Lyons, W. A., and Nelson, T.: Observations of columniform sprites, J. Atmos. Solar-Terr. Phys., 60, 733-740, 1998.

Wescott, E. M., Sentman, D. D., Stenbaek-Nielsen, H. C., Huet, P., Heavner, M. J., and Moudry, D. R.: New evidence for the brightness and ionization of blue starters and blue jets, J. Geophys. Res., 106, 21549-21554, 2001.

Williams, E., Downes, E., Boldi, R., Lyons, W., and Heckman, S.: Polarity asymmetry of sprite-producing lightning: A paradox?, Radio Sci., 42, RS2S17, doi:10.1029/2006RS003488, 2007.

Wilson, C. T. R.: The electric field of a thundercloud and some of its effects, Proc. Phys. Soc. London, 37, 32D-37D, 1925.

Winckler, J., Lyons, W., Nelson, T., and Nemzek, R.: New highresolution ground-based studies of sprites, J. Geophys. Res., 101, 6997-7004, 1996.

Yair, Y., Israelevich, P., Devir, A. D., Moalem, M., Price, C., Joseph, J. H., Levin, Z., Ziv, B., Sternlieb, A., and Teller, A.: New observations of sprites from space shuttle, J. Geophys. Res., 109, D15201, doi:10.1029/2003JD004497, 2004.

Yashunin, S. A., Mareev, E. A., and Rakov, V. A.: Are lightning M components capable of initiating sprites and sprite halo?, J. Geophys. Res., 112, D10109, doi:10.1029/2006JD007631, 2007.

Yukhimuk, V., Roussel-Dupré, R., Symbalisty, E. M. D., and Taranenko, Y.: Optical characteristics of Red Sprites produced by runaway air breakdown, J. Geophys. Res., 103, 11473-11482, 1998.

Yukhimuk, V., Roussel-Dupré, R., and Symbalisty, E. M. D.: On the temporal evolution of red sprites: Runaway theory versus data, Geophys. Res. Lett., 26, 679-682, 1999. 